\title{
Local signature defect of fibered complex surfaces via monodromy and stable reduction
}

Tadashi Ashikaga

\begin{abstract}
We consider the localization problem of signature of a fibered complex surface. In this paper, we analyze the contribution of topological monodromy to the local signature of a fiber germ. In order to realize it, we describe explicitly the behavior of Atiyah-Patodi-Singer's eta invariant under the stable reduction of a fiber germ in terms of Nielsen's data.
\end{abstract}

Mathematics Subject Classification (2000). 14D06, 14H15, 14J80, 32Q55, 57M50, 57M60.

Keywords. Signature, fibered surface, monodromy, eta invariant, stable reduction, signature defect.

\section{Introduction}

Let $\boldsymbol{f}: \boldsymbol{S} \rightarrow \boldsymbol{B}$ be a surjective holomorphic map from a compact complex surface $\boldsymbol{S}$ to a nonsingular curve $\boldsymbol{B}$ such that the general fiber of $\boldsymbol{f}$ is a curve of genus $g$. We call $\boldsymbol{f}$ a fibration of genus $g$. Let Sign $\boldsymbol{S}$ be the signature of the intersection form on $H^{2}(\boldsymbol{S}, \mathbb{Q})$. If there exist a finite number of fiber germs $\left(f, F_{i}\right), F_{i}=\boldsymbol{f}^{-1}\left(P_{i}\right)$ $\left(1 \leq i \leq s, P_{i} \in \boldsymbol{B}\right)$ and a local invariant $\sigma\left(f, F_{i}\right)$ is geometrically well defined such that

$$
\operatorname{Sign} S=\sum_{i=1}^{s} \sigma\left(f, F_{i}\right),
$$

then we say $\operatorname{Sign} S$ is localized and call $\sigma\left(f, F_{i}\right)$ a local signature.

Atiyah [At] had a deep insight which is, in some sense, the origin of several formulations of this concept. Matsumoto [Ma1], [Ma2] used the Meyer function and formulated the local signature of genus 1 and 2, and calculated them for the Lefschetz fiber germs. Endo [E] extended it for hyperelliptic fibrations of arbitrary genus. (See also [Mo].) Another local signature for hyperelliptic fibrations is defined by using the double covering method ( [X], [AA1]), and these two notions are in fact coincide with each other ([Te2]).

On the other hand, Ueno [U] used the even theta constant and defined and calculated a local signature of genus 2. Iida [Ii] gave an analytic interpretation of the local 
signature of genus 2 by using the theta divisor and the adiabatic limit of the eta invariant. Kuno $[\mathrm{Ku}]$ extended the approach of the Meyer function to non-hyperelliptic fibrations of genus 3 . For the survey articles, see [AK], [AE].

Now, through a series of papers, we present a certain type of a local signature including generic non-hyperelliptic and unstable fibrations of arbitrary genus. From our viewpoint, this notion should philosophically consist of two aspects of "moduli" and "monodromy":

$$
\text { local signature := "moduli" aspect + "monodromy" aspect. }
$$

With respect to the moduli aspect, we refer the joint paper with K. Yoshikawa [AY] in detail. Here we only comment that, if $\boldsymbol{f}: \boldsymbol{S} \rightarrow \boldsymbol{B}$ is a stable fibration, then the moduli aspect of (1) purely contribute it. Namely, by developing the idea of I. Smith [Sm], we can define an explicit "signature divisor" on the Deligne-Mumford compactification $\bar{M}_{g}$ and can localize Sign $S$ via the pull back of it by the induced map $\boldsymbol{B} \rightarrow \bar{M}_{g}$.

The main topic of this paper is the analysis of the monodromy aspect of (1), which concerns the direct relation between the local monodromy around the singular fibers and the local contribution of the stable reduction to the global signature. Namely, our purpose here is to write down it explicitly by the language of the Nielsen's invariants [N1], [N2] of the monodromy map.

Note that Tan [Tan] and Viehweg [V] had important studies on the contribution of the stable reduction to global invariants of $\boldsymbol{S}$ by certain algebro-geometric methods. On the other hand, our method is topological based on variations of the signature theorem historically started from [Hi1], and the setting is local in base.

We summarize the arguments here. Let $f: S \rightarrow \Delta$ be a degeneration of curves with the central fiber $F$ over a closed disk $\Delta$, and let $h_{S}$ be a Riemannian metric on $S$ such that $h_{S}$ is a product metric near the boundary $\partial S$. We define

$$
\sigma\left(f, F ; h_{S}\right)=\operatorname{Sign} S+\eta\left(\partial S, h_{\partial S}\right),
$$

where Sign $S$ is the signature on $H^{2}(S, \partial S ; \mathbb{Q})$ and $\eta\left(\partial S, h_{\partial S}\right)$ is the eta invariant ([APS]) with respect to the restricted metric $h_{\partial S}$ of $h_{S}$ on the boundary $\partial S$. Let $\tilde{f}: \widetilde{S} \rightarrow \widetilde{\Delta}$ be the minimal stable reduction of $f$ with the central fiber $\widetilde{F}$ which comes from the cyclic base change $\widetilde{\Delta} \rightarrow \Delta$ of degree $N$. We also put $\sigma\left(\tilde{f}, \widetilde{F} ; h_{\partial \widetilde{S}}\right)=$ Sign $\widetilde{S}+\eta\left(\partial \widetilde{S}, h_{\partial \widetilde{S}}\right)$ where $h_{\partial \widetilde{S}}$ is a Riemannian metric on $\partial \widetilde{S}$ which coincides with the natural pull back of $h_{\partial \tilde{S}}$. We define

$$
\operatorname{Lsd}\left(f, F ; h_{\partial S}\right):=\sigma\left(f, F ; h_{\partial S}\right)-\frac{1}{N} \sigma\left(\tilde{f}, \widetilde{F} ; h_{\partial \widetilde{S}}\right),
$$

which we call the local signature defect of the degeneration $f$. This notion is analogous in some sense to the "defect part" which Hirzebruch explained in [Hi2]. 
Now our main theorem (Theorem 5.2.1) says that the local signature defect of $f$ is explicitly written in terms of the Nielsen's monodromy data of $f$. In particular, this is independent of the choice of the metric $h_{\partial S}$. Moreover the local signature defect is nothing but the local contribution of the difference of the global signatures for the global stable reduction (Lemma 5.3.1).

The idea of the proof of the main theorem is as follows: The cyclic group $G=$ $\mathbb{Z} /(N)$ acts holomorphically on $\widetilde{S}$ so that the resolution space of the singularities of the quotient space $\widetilde{S} / G$ coincides with the original $S$, and the problem is reduced to the comparison between Sign $\widetilde{S}$ and Sign $\widetilde{S} / G$. Since the spaces $\widetilde{S}$ and $\widetilde{S} / G$ are also complex orbifolds, we can apply Kawasaki's orbifold signature theorem [Ka], which is a natural extension of Atiyah-Singer's $G$-signature theorem. The data of the integral of the equivariant L-forms on the infinitesimal neighborhoods of the $G$-fixed point sets are essentially reduced to the Dedekind sum with respect to the monodromy data, which are calculated explicitly in Sections 4 and 6.

For the preparation of this calculation, we propose the following two tools, which themselves seem to have independent meanings. The one is a formula of Dedekind sum (Theorem 4.1.2), which is due to Myerson and Holzapfel ([Hol], [My]). The other is a certain precise relation between the stable reduction and the MatsumotoMontesinos' method in Sections 1-3 as follows:

The study of the local monodromy has a long history, and it is settled that the local monodromy as an element in the conjugacy class of the mapping class group of genus $g$ belongs to the class of pseudo-periodic map of negative twist. Conversely, for a given pseudo-periodic map of negative twist $\mu: \Sigma_{g} \rightarrow \Sigma_{g}$ of a Riemann surface $\Sigma_{g}$, Matsumoto-Montesinos ([MM1] Part I) constructed a certain topological quotient space $\Sigma_{g} \rightarrow \Sigma_{g} /\langle\mu\rangle$, which we call MM-quotient.

An algebro-geometric interpretation of $\Sigma_{g} /\langle\mu\rangle$ due to Takamura [Tak] is as follows: We start from the stable reduction $\widetilde{S} \rightarrow \widetilde{\Delta}$ of $f$. The Galois group $G=\operatorname{Gal}(\widetilde{\Delta} / \Delta)$ acts holomorphically on $\widetilde{S}$, since it is 2-dimensional ([DM]). The $G$ action has natural local expressions at the nodes and the points whose isotropy groups are nontrivial as in Section 3.1 (cf. [Te1]). He constructed the local uniformization spaces of these actions and determined the types of singularities on $\widetilde{S} / G$. The normally minimal model of the resolution of $\widetilde{S} / G$ is nothing but the space $\Sigma_{g} /\langle\mu\rangle$. Therefore, to say generically, the stable reduction theorem induce the MM-quotient.

The MM-quotient has richer information than the usual stable reduction theorem. Indeed, we propose in Section 2 "a precise" stable reduction theorem based on MMquotient as follows: Since the numerical Chorizo space of the singular fiber of $f$ coincides with $\Sigma_{g} /\langle\mu\rangle$, all the irreducible components are classified into cores, tails, arcs and quasi-tails as in Section 1. We contract all the tails, arcs and quasi-tails to points, and put $S^{\#}$ the resulting surface. Then the natural fibration $\widetilde{S} \rightarrow \widetilde{\Delta}$ of the normalization $\widetilde{S}$ of $S^{\sharp} \times_{\Delta} \widetilde{\Delta}$ via the cyclic cover $\widetilde{\Delta} \rightarrow \Delta$ whose degree coincides with a pseudo-period of $\mu$ is nothing but the stable reduction of $f$ (Theorem 2.2.1). This 
process is more precise than the usual one, because the birational transformations in the process are explicit. Moreover, the description of the Milnor number of the A-type singularities on $\widetilde{S}$, the coincidence of $S^{\#}$ and $\widetilde{S} / G$, and the description of the singularities on $S^{\sharp}$ are all directly known.

By comparing the monodromy maps of $\widetilde{S} \rightarrow \widetilde{\Delta}$ and $\widetilde{S} / G \rightarrow \Delta=\widetilde{\Delta} / G$, we also directly obtain the coincidence of the data of the local action of $G$ to $\widetilde{S}$ (which we call $G$-valency etc. in Section 3.1) and the Nielsen's monodromy data (Theorem 3.1.1).

\section{Matsumoto-Montesinos quotient}

In this section, we review the construction of the quotient space introduced by Matsumoto-Montesinos ([MM1] Part I) and related facts which will be used afterwards. ${ }^{1}$ This space is constructed by the topological quotient of a Riemann surface by an element of the subclass of the mapping class group which is called pseudo-periodic map of negative twist. Here our description is slightly modified from the original one [MM1] by using the method of Takamura [Tak].

1.1. Let $\Sigma_{g}$ be a Riemann surface of genus $g$ and let $\mu: \Sigma_{g} \rightarrow \Sigma_{g}$ be a pseudoperiodic map of negative twist. By definition, $\mu$ is an orientation-preserving homeomorphism such that the following conditions are satisfied modulo isotopy: There exists a decomposition

$$
\Sigma_{g}=\boldsymbol{A} \cup \boldsymbol{B}
$$

into the annulus-part $\boldsymbol{A}=\coprod \mathcal{A}_{j}$ which is the disjoint union of the annular neighborhoods $\mathcal{A}_{j}$ of simple closed curves on $\Sigma_{g}$ belonging to the admissible system of cut curves, and the body-part $\boldsymbol{B}=\bigsqcup B_{i}$ which is the disjoint union of Riemann surfaces $B_{i}$ with boundary, such that the boundary set $\partial \boldsymbol{B}$ coincides with the boundary set $\partial \boldsymbol{A}$. The restriction $\left.\mu\right|_{\boldsymbol{B}}$ is periodic, i.e., the power $\left(\left.\mu\right|_{\boldsymbol{B}}\right)^{N}$ for some natural number $N$ is the identity map $\operatorname{id}_{\boldsymbol{B}}$. The restriction of the power $\left(\left.\mu\right|_{\mathcal{A}_{j}}\right)^{N}$ to each annulus $\mathcal{A}_{j}$ is a right-handed integral Dehn twist. We call such an $N$ a pseudo-period of $\mu$. Note that $N$ is a multiple of the minimal pseudo-period $N_{0}$ (i.e., the minimal natural number among all the pseudo-periods).

Now let $[\mu]$ be the equivalence class of $\mu$ of the conjugacy class $\widehat{\mathcal{M}}_{g}$ of the mapping class group of genus $g$. We review the conjugacy invariants of $[\mu]$ :

Let $\vec{C}$ be an oriented simple closed curve on $\Sigma_{g}$. Suppose there is a natural number $m=m(\vec{C})$ such that $\mu^{m}(\vec{C})=\vec{C}$ as an oriented curve, where $m$ is assumed to be the minimal number which enjoys this property. Moreover suppose $(\mu \mid \vec{C})^{m}$ is periodic of order $\lambda=\lambda(\vec{C}) \geq 1$. Then for any point $R$ on $\vec{C}$, there is a natural number $\sigma=\sigma(\vec{C})$ with $1 \leq \sigma \leq \lambda-1$ such that the iteration of $\mu^{m}$ are situated in the

\footnotetext{
${ }^{1}$ For many examples of the notion discussed here, see for instance [AI1].
} 
order $\left(R, \mu^{m \sigma}(R), \mu^{2 m \sigma}(R), \ldots, \mu^{(\lambda-1) m \sigma}(R)\right)$ when viewed in the direction of $\vec{C}$. The set of triple $(m(\vec{C}), \lambda(\vec{C}), \sigma(\vec{C}))$ is called Nielsen's valency at $\vec{C}$ ([N1]). Let $\delta=\delta(\vec{C})$ be the integer which satisfies $\sigma \delta \equiv 1(\bmod \lambda)$ and $1 \leq \delta \leq \lambda-1$. Then the map $\left.\mu^{m}\right|_{\vec{C}}$ behaves as the rotation of angle $2 \pi \delta / \lambda$ in a suitable parametrization of $\vec{C}$. Since the number $\delta$ is also important in our argument, we simply call the quadruplet $(m(\vec{C}), \lambda(\vec{C}), \sigma(\vec{C}), \delta(\vec{C}))$ the valency at $\vec{C}$.

Let $Q$ be a point on the inside of a body component $B_{i} \backslash \partial B_{i}$. Let $m\left(B_{i}\right)$ be the minimal natural number such that $\left.\mu^{m\left(B_{i}\right)}\right|_{B_{i}}=\mathrm{id}_{B_{i}}$. If there exists a natural number $m=m(Q)$ which is strictly smaller than $m\left(B_{i}\right)$ such that the points $\left\{Q, \mu(Q), \ldots, \mu^{m-1}(Q)\right\}$ are distinct each other and $\mu^{m}(Q)=Q$, we call $Q$ a multiple point. Then there exists a disk neighborhood $D_{Q}$ of $Q$ which is invariant under the action $\mu^{m}$. The valency $(m(Q), \lambda(Q), \sigma(Q), \delta(Q))$ at $Q$ is defined to be the valency of the boundary curve $\partial D_{Q}$ of $D_{Q}$ whose orientation is defined from the outside of the disk. (Note that the orientation of $\partial D_{Q}$ is defined from the inside in [MM1].)

For an annulus component $\mathcal{A}_{j}$, we put $\partial \mathcal{A}_{j}=\partial \mathcal{A}_{j}^{(1)} \amalg \partial \mathcal{A}_{j}^{(2)}$ the decomposition to connected components of the boundary curve where the orientation is defined here from the outside of the annulus. The valency at $\mathcal{A}_{j}$ is defined to be the couple of valencies $\left(m\left(\partial \mathcal{A}_{j}^{(k)}\right), \lambda\left(\partial \mathcal{A}_{j}^{(k)}\right), \sigma\left(\partial \mathcal{A}_{j}^{(k)}\right), \delta\left(\partial \mathcal{A}_{j}^{(k)}\right)\right)$ for $k=1,2$. If there exists a natural number $\beta$ such that $\mu^{\beta}$ interchanges the boundary components of $\mathscr{A}_{j}$, i.e., $\mu^{\beta}\left(\partial \mathcal{A}_{j}^{(1)}\right)=\partial \mathcal{A}_{j}^{(2)}$, we call $\mathcal{A}_{j}$ an amphidrome annulus. Otherwise we call $\mathcal{A}_{j}$ a non-amphidrome annulus.

Let $\alpha$ be the smallest natural number such that $\mu^{\alpha}\left(\mathcal{A}_{j}\right)=\mathcal{A}_{j}$ does not interchange the boundary components. Let $\gamma$ be a non-zero integer such that $\left.\mu^{\gamma}\right|_{\partial \mathcal{A}_{j}}$ is the identity map. Then $\gamma$ is a multiple of $\alpha$, and $\mu^{\gamma}: \mathcal{A}_{j} \rightarrow \mathcal{A}_{j}$ is a result of $e$ full Dehn twist, $e$ being an integer. Then we define the screw number at $\mathcal{A}_{j}$ by $s\left(\mathcal{A}_{j}\right):=e \alpha / \gamma$.

Then the theorem of Nielsen [N2] and Matsumoto-Montesinos [MM1] says that the conjugacy class of $\mu$ is determined by the data of

(i) valencies at the multiple points $\{Q\}$ and at the annuli $\left\{\mathcal{A}_{j}\right\}$,

(ii) screw numbers at the annuli $\left\{\mathcal{A}_{j}\right\}$,

(iii) the action of $\mu$ to the extended partition graph $\Gamma(\mu)$, i.e., the one-dimensional oriented graph whose points correspond to $\left\{B_{i}\right\}$ and whose segments correspond to $\left\{\mathcal{A}_{j}\right\}$ in a natural way.

1.2. By a numerical Chorizo space, we mean a connected topological space consisting of the components which are underlying topological spaces of irreducible Riemann surfaces with nodes so that the multiplicities are attached to every components. Moreover, if two components of them intersect each other, they intersect at several points transversally. 
For a given conjugacy class of pseudo-periodic map of negative twist $[\mu]$, Matsumoto-Montesinos [MM1] constructed the generalized quotient map $\pi_{\mu}: \Sigma_{g} \rightarrow$ $\Sigma_{g} /\langle\mu\rangle$. Here $\Sigma_{g} /\langle\mu\rangle$ is a certain numerical Chorizo space, which we call the Matsumoto-Montesinos quotient of $\mu$. Let $\Sigma_{g} /\langle\mu\rangle=\sum_{i} \alpha_{i} F_{\text {top }}^{(i)}$ be the decomposition to its components. By the construction, $F_{\text {top }}^{(i)}$ is classified into the following types (i)-(iv):

(i) $F_{\text {top }}^{(i)}$ is a core component. Namely, $F_{\text {top }}^{(i)}$ is the unique component which contains the support of $\pi_{\mu}\left(B_{i}^{\prime}\right)$, where $B_{i}^{\prime}$ is the complement of disk neighborhoods of multiple points of $\mu$ on a certain body connected component $B_{i}$,

(ii) $F_{\text {top }}^{(i)}$ is a sphere component of a tail which comes from the quotient of a disk neighborhood of a multiple point,

(iii) $F_{\text {top }}^{(i)}$ is a sphere component of an $\operatorname{arc}$ which comes from the quotient of a nonamphidrome annulus,

(iv) $F_{\text {top }}^{(i)}$ is a sphere component of a quasi-tail which comes from the quotient of an amphidrome annulus.

We review the construction and the properties of the tails, the arcs and the quasitails in Sections 4, 1.5 and 1.6, respectively.

1.3. Before the construction, we prepare some terminology. Let $(\lambda, \sigma)$ be any pair of integers with $1 \leq \sigma \leq \lambda-1$ in this subsection. Let

$$
\frac{\lambda}{\sigma}=K_{1}-\frac{n_{2}}{\sigma}=K_{1}-\frac{1}{K_{2}-\frac{n_{3}}{n_{2}}}=K_{1}-\frac{1}{K_{2}-\frac{1}{K_{3}-\cdots}}:=\left[\llbracket K_{1}, K_{2}, \ldots, K_{r} \rrbracket\right]
$$

be the continued linear fraction. The sequence $\left\{n_{i}\right\}_{i=0}^{r+1}$ of natural numbers which satisfies $n_{0}=\lambda, n_{1}=\sigma$ and

$$
n_{i}=K_{i-1} n_{i-1}-n_{i-2}(2 \leq i \leq r+1)
$$

is called the usual multiplicity sequence of the continued linear fraction (3). Note that $n_{r}=1$ and $n_{r+1}=0$.

Conversely, let $\left\{n_{i}\right\}_{i=0}^{r+1}$ be any sequence of non-negative integers such that $n_{i} \neq 0$ and $\left(n_{i+1}+n_{i-1}\right) / n_{i}$ are integers for $1 \leq i \leq r$. We call such $\left\{n_{i}\right\}_{i=0}^{r+1}$ abstract multiplicity sequence. We define the associated continued fraction $\left[\left[K_{1}, K_{2}, \ldots, K_{r}\right]\right.$ of this abstract multiplicity sequence by putting $K_{i}=\left(n_{i+1}+n_{i-1}\right) / n_{i}$.

For instance, we sometimes set the initial values $n_{0}, n_{1}$ another pair of natural numbers, and consider the abstract multiplicity sequence by the same recursion formula (4) of (3) as in the following example. 
Example 1.3.1. We consider $\left[\left[K_{1}, K_{2}, K_{3}, K_{4}, K_{5}\right]\right]=\llbracket[2,2,2,3,2] \rrbracket=14 / 11$. Then the usual multiplicity sequence is $\{14,11,8,5,2,1,0\}$.

On the other hand, the abstract multiplicity sequence with the initial values $n_{0}=6$, $n_{1}=5$ of this continued linear fraction is $\{6,5,4,3,2,3,4\}$. Indeed, we have $n_{2}=2 \cdot 5-6=4, n_{3}=2 \cdot 4-5=3, n_{4}=2 \cdot 3-4=2, n_{5}=3 \cdot 2-3=3$, $n_{6}=2 \cdot 3-2=4$.

Therefore, [[2, 2, 2, 3,2]] is also the associated continued linear fraction of $\{6,5,4,3,2,3,4\}$.

1.4. Let $Q$ be a multiple point on $\boldsymbol{B}$ with the valency $(m, \lambda, \sigma, \delta)$. Let (3) be the continued linear fraction of $\lambda / \sigma$ with respect to this valency data, and let $\left\{n_{i}\right\}_{i=0}^{r+1}$ be its usual multiplicity sequence.

Then we attach at a point of a disk $\mathcal{D}$ of multiplicity $m n_{0}$ a chain of rational curves of the length $r$ of multiplicities $m n_{1}, m n_{2}, \ldots, m n_{r}$ (see [MM2], p. 72, Figure 1). This is the tail arising from the multiple point $Q$.

1.5. Next consider a non-amphidrome annulus $\mathcal{A}=\mathcal{A}_{j}$. Let $\partial \mathcal{A}=\partial \mathcal{A}^{(1)} \bigsqcup \partial \mathcal{A}^{(2)}$ be the decomposition to the connected components of the boundary and let $\left(m^{(k)}, \lambda^{(k)}, \sigma^{(k)}, \delta^{(k)}\right)$ be the valencies at $\partial \mathcal{A}^{(k)}(k=1,2)$. Let $s(\mathcal{A})$ be the screw number at $\mathcal{A}$. Then $s(\mathcal{A})$ is a non-positive rational number, and is written as

$$
s(\mathcal{A})=-\frac{\delta^{(1)}}{\lambda^{(1)}}-\frac{\delta^{(2)}}{\lambda^{(2)}}-K
$$

where $\boldsymbol{K}$ is an integer greater or equal to -1 ([MM1]). Let

$$
\frac{\lambda^{(1)}}{\sigma^{(1)}}=\left[\left[K_{1}, K_{2}, \ldots, K_{r}\right]\right], \quad \frac{\lambda^{(2)}}{\sigma^{(2)}}=\left[\left[L_{1}, L_{2}, \ldots, L_{r^{\prime}}\right]\right]
$$

be the continued linear fractions and let $\left\{n_{i}\right\}_{i=0}^{r+1},\left\{m_{i}\right\}_{i=0}^{r^{\prime}+1}$ be their usual multiplicity sequences respectively. Let $\hat{\sigma}^{(i)}$ be the natural number which satisfies

$$
\sigma^{(i)} \delta^{(i)}=\hat{\sigma}^{(i)} \lambda^{(i)}+1 \quad(i=1,2) .
$$

We define a natural number $d$ and integers $v, v^{*}, \bar{v}, \bar{v}^{*}$ by

$$
\begin{gathered}
d=-\lambda^{(1)} \lambda^{(2)} s(\mathcal{A})=\delta^{(1)} \lambda^{(2)}+\delta^{(2)} \lambda^{(1)}+\boldsymbol{K} \lambda^{(1)} \lambda^{(2)} \\
v=\sigma^{(1)} \delta^{(2)}+\lambda^{(2)} \hat{\sigma}^{(1)}+\sigma^{(1)} \lambda^{(2)} \boldsymbol{K}, \quad v^{*}=\sigma^{(2)} \delta^{(1)}+\lambda^{(1)} \hat{\sigma}^{(2)}+\sigma^{(2)} \lambda^{(1)} \boldsymbol{K}, \\
\bar{v} \equiv v(\bmod d), 1 \leq \bar{v} \leq d-1, \quad \bar{v}^{*} \equiv v^{*}(\bmod d), 1 \leq \bar{v}^{*} \leq d-1 .
\end{gathered}
$$

Lemma 1.5.1 (Takamura [Tak]). (i) $v v^{*} \equiv 1(\bmod d)$,

(ii) If $\boldsymbol{K} \geq 0$, then $1 \leq v \leq d-1$ and $1 \leq v^{*} \leq d-1$, i.e., $v=\bar{v}$ and $v^{*}=\bar{v}^{*}$. 
Proof. The assertion (i) follows from

$$
v v^{*}=\left(\hat{\sigma}^{(1)} \sigma^{(2)}+\sigma^{(1)} \hat{\sigma}^{(2)}+\sigma^{(1)} \sigma^{(2)} \boldsymbol{K}\right) d+1 .
$$

The former assertion of (ii) follows from

$$
d-v=\frac{\left(\lambda^{(1)}-\sigma^{(1)}\right) d+\lambda^{(2)}}{\lambda^{(1)}}>0 .
$$

The latter assertion of (ii) is similar.

Now we first assume $\boldsymbol{K} \geq 0$. From (6), we define new continued fractions $\Phi:=$ $\Phi\left(\lambda^{(1)} / \sigma^{(1)}, \lambda^{(2)} / \sigma^{(2)}, \boldsymbol{K}\right)$ as follows:

(i) If $\boldsymbol{K} \geq 1$, put

$$
\Phi=\left[[K_{1}, K_{2}, \ldots, K_{r-1}, K_{r}+1, \underbrace{2, \ldots, 2}_{\boldsymbol{K}-1}, L_{r^{\prime}}+1, L_{r^{\prime}-1} \ldots, L_{2}, L_{1}]\right] .
$$

(ii) If $\boldsymbol{K}=0, r \geq 1$ and $r^{\prime} \geq 1$, put

$$
\Phi=\llbracket\left[K_{1}, K_{2}, \ldots, K_{r-1}, K_{r}+L_{r^{\prime}}, L_{r^{\prime}-1} \ldots, L_{2}, L_{1} \rrbracket\right] .
$$

(iii) If $\boldsymbol{K}=0, r \geq 1$ and $r^{\prime}=0$, put

$$
\Phi=\left[\left[K_{1}, K_{2}, \ldots, K_{r-1}\right]\right] .
$$

Lemma 1.5.2. The abstract multiplicity sequences with the initial values $\lambda^{(1)}, \sigma^{(1)}$ of the continued linear fraction $\Phi$ are as follows:

(i) If $\boldsymbol{K} \geq 1$, then $\{n_{0}, n_{1}, \ldots, n_{r-1}, n_{r}, \underbrace{1, \ldots, 1}_{\boldsymbol{K}-1}, m_{r^{\prime}}, m_{r^{\prime}-1}, \ldots, m_{1}, m_{0}\}$.

(ii) If $\boldsymbol{K}=0, r \geq 1$ and $r^{\prime} \geq 1$, then $\left\{n_{0}, n_{1}, \ldots, n_{r-1}, 1, m_{r^{\prime}-1}, \ldots, m_{1}, m_{0}\right\}$.

(iii) If $\boldsymbol{K}=0, r \geq 1$ and $r^{\prime}=0$, then $\left\{n_{0}, n_{1}, \ldots, n_{r-1}, n_{r}\right\}$.

Proof. We prove (i). Let $\left\{\ell_{i}\right\}_{i=0}^{r+r^{\prime}+\boldsymbol{K}}$ be the abstract multiplicity sequence of $\Phi$ with the initial values $\ell_{0}=\lambda^{(1)}=n_{0}, \ell_{1}=\sigma^{(1)}=n_{1}$. It is clear that $\ell_{i}=n_{i}$ for $0 \leq i \leq r$. Since $n_{r}=1$ and $K_{r} n_{r}-n_{r-1}=0$, we also have $\ell_{r+1}=$ $\left(K_{r}+1\right) n_{r}-n_{r-1}=1$ and $\ell_{r+i}=2 \cdot 1-1=1$ for $2 \leq i \leq \boldsymbol{K}$.

On the other hand, the usual multiplicity sequence $\left\{m_{i}\right\}_{i=0}^{r^{\prime}+1}$ satisfies $m_{r^{\prime}+1}=0$, $m_{r^{\prime}}=1$ and $m_{i-2}=L_{i-1} m_{i-1}-m_{i}$ for $2 \leq i \leq r^{\prime}+1$. Hence

$$
\ell_{\boldsymbol{r}+\boldsymbol{K}+1}=1 \cdot\left(L_{\boldsymbol{r}^{\prime}}+1\right)-1=L_{\boldsymbol{r}^{\prime}}=m_{\boldsymbol{r}^{\prime}-1} .
$$

By (10) and $\ell_{\boldsymbol{r}+\boldsymbol{K}}=1=m_{\boldsymbol{r}^{\prime}}$, we inductively have

$\ell_{\boldsymbol{r}+\boldsymbol{K}+i}=L_{\boldsymbol{r}^{\prime}-i-1} \ell_{\boldsymbol{r}+\boldsymbol{K}+i-1}-\ell_{\boldsymbol{r}+\boldsymbol{K}+i-2}=L_{\boldsymbol{r}^{\prime}-i-1} m_{\boldsymbol{r}^{\prime}-i+1}-m_{\boldsymbol{r}^{\prime}-i+2}=m_{\boldsymbol{r}^{\prime}-i}$ for $2 \leq i \leq r^{\prime}$.

The proofs of (ii), (iii) are similar. 
Lemma 1.5.3. The value of the continued linear fraction of each of $\Phi$ coincides with $d / v$.

Proof. See [To] p. 64, Lemma 4.9. Note that Lemma 1.5 .3 also implies that $\left[[L_{1}, L_{2}, \ldots, L_{r^{\prime}-1}, L_{r^{\prime}}+1, \underbrace{2, \ldots, 2}_{\boldsymbol{K}-1}\right.$,
$\left.\left.K_{r}+1, K_{r-1} \ldots, K_{2}, K_{1}\right]\right]=d / v^{*}$ and so on.

Next we assume $\boldsymbol{K}=-1$. This case occur only when $\delta^{(1)} / \lambda^{(1)}+\delta^{(2)} / \lambda^{(2)} \geq 1$.

Theorem 1.5.4 (Takamura). Assume $\delta^{(1)} / \lambda^{(1)}+\delta^{(2)} / \lambda^{(2)} \geq 1$.

(i) There exists an unique pair $\left(\omega, \omega^{\prime}\right)$ of integers with $0 \leq \omega \leq r, 0 \leq \omega^{\prime} \leq r^{\prime}$, $\left(\omega, \omega^{\prime}\right) \neq(0,0)$ which satisfies

$$
n_{\omega}=m_{\omega^{\prime}}, \quad n_{\omega+1}+m_{\omega^{\prime}+1}=n_{\omega} .
$$

In particular, the following are abstract multiplicity sequences;

(a) If $\omega \geq 1, \omega^{\prime} \geq 1$, then $\left\{n_{0}, n_{1}, \ldots, n_{\omega-1}, n_{\omega}, m_{\omega^{\prime}-1}, \ldots, m_{1}, m_{0}\right\}$.

(b) If $\omega^{\prime}=0$, then $\left\{n_{0}, n_{1}, \ldots, n_{\omega-1}, n_{\omega}\right\}$.

(ii) The associated continued linear fractions $\Phi$ of these sequences are respectively

(a) $\Phi=\left[\left[K_{1}, K_{2}, \ldots, K_{\omega-1}, K_{\omega}+L_{\omega^{\prime}}-1, L_{\omega^{\prime}-1}, \ldots, L_{2}, L_{1}\right]\right.$,

(b) $\Phi=\left[\left[K_{1}, K_{2}, \ldots, K_{\omega-1}\right]\right]$ for $\omega \geq 2$. (Note that $\Phi$ is empty for $\omega=1$.)

(iii) In the case (a), we have $v=\bar{v}$ and $v^{*}=\bar{v}^{*}$. Moreover the value of the continued linear fraction $\Phi$ coincide with $d / v$ by putting $\boldsymbol{K}=-1$ in (8).

(iv) In the case (b) for $\omega \geq 2$, we have $v<\bar{v}$ and $v^{*}=\bar{v}^{*}$. The value of $\Phi$ coincide with $d / \bar{v}$ by putting $\boldsymbol{K}=-1$.

Proof. See [Tak], §6.2.

We will again discuss in Section 3.2 the geometric meaning of Lemma 1.5.3 and Theorem 1.5.4.

Now we go back to the construction of the $\operatorname{arc} \pi_{\mu}(\mathcal{A})$. This is related to the abstract multiplicity sequences in Lemma 1.5.2 and Theorem 1.5.4. Namely, this is constructed by combining two disks $\mathscr{D}^{(1)}$ and $\mathscr{D}^{(2)}$ with multiplicities $m n_{0}$ and $m m_{0}$ respectively by the following chain of spheres:

(i) If $\boldsymbol{K} \geq 1$, then the chain has length $r+r^{\prime}+K-1$ such that the multiplicities of the components are $m n_{1}, m n_{2}, \ldots, m n_{r}, \underbrace{m, \ldots, m}, m m_{r^{\prime}}, \ldots, m m_{2}, m m_{1}$ (see [MM2], p. 73, Figure 2).

$$
\text { K }-1
$$

(ii) If $\boldsymbol{K}=0$, then the chain has length $r+r^{\prime}-1$ such that the multiplicities of the components are $m n_{1}, m n_{2}, \ldots, m n_{r-1}, m, m m_{r^{\prime}-1}, \ldots, m m_{2}, m m_{1}$ (Figure A). 
(iiia) If $\boldsymbol{K}=-1, \omega \geq 1$ and $\omega^{\prime} \geq 1$, then the chain has length $\omega+\omega^{\prime}-1$ such that the multiplicities of the components are $m n_{1}, m n_{2}, \ldots, m n_{\omega-1}, m n_{\omega}, m m_{\omega^{\prime}-1}, \ldots$, $m m_{2}, m m_{1}$ (Figure B).

(iiib) If $\boldsymbol{K}=-1, \omega \geq 2$ and $\omega^{\prime}=0$, then the chain has length $\omega-1$ such that the multiplicities of the components are $m n_{1}, m n_{2}, \ldots, m n_{\omega-1}$ (Figure C).

(iiic) If $\boldsymbol{K}=-1$ and $\omega=1, \omega^{\prime}=0$, then the banks of both sides of the bodies connect directly at one point transversally (Figure D).
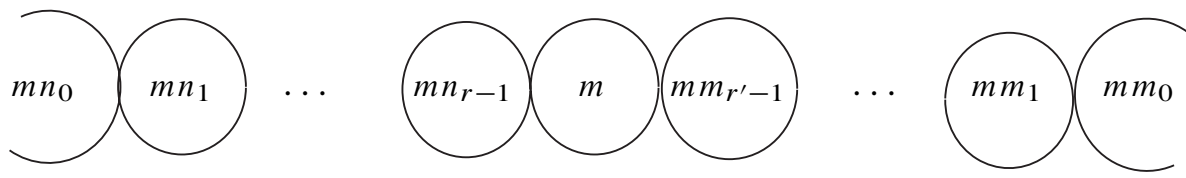

Figure A
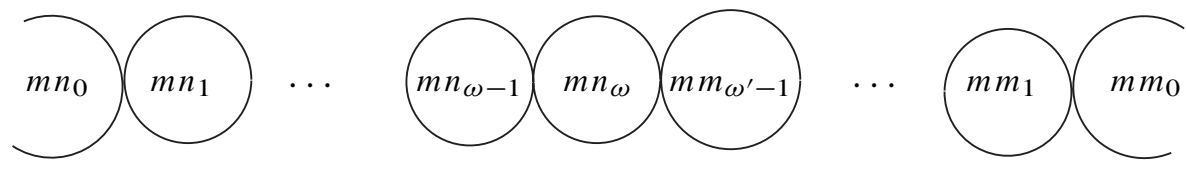

Figure B

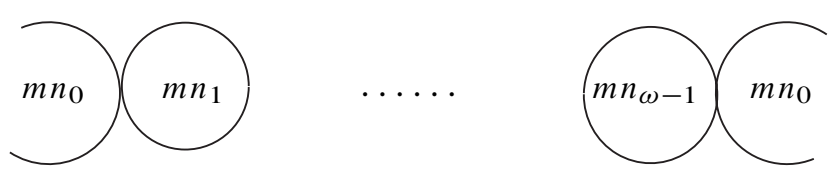

Figure C



Figure D

The above $\operatorname{arc} \pi_{\mu}(\mathcal{A})$ is globally attached to core components in a natural way. 
1.6. Lastly we assume that the annulus $\mathcal{A}$ is amphidrome. The quasi-tail $\pi_{\mu}(\mathcal{A})$ is constructed as follows: Let $\left(2 m^{\prime}, \lambda, \sigma, \delta\right)$ be the valency at both sides of boundary curves of $\mathcal{A}$, and let $\left\{n_{i}\right\}_{i=0}^{r}$ be the multiplicity sequence of the continued linear fraction of $\lambda / \sigma$ as in (3). Then $\pi_{\mu}(\mathcal{A})$ is constructed by connecting a disk of multiplicity $2 m^{\prime} m_{0}$ and the set of rational curves at a point transversally. This set has the dual graph of Dynkin diagram of type $\mathrm{D}$, and the multiplicities of components are $2 m^{\prime} n_{1}, 2 m^{\prime} n_{2}, \ldots, 2 m^{\prime} n_{r}, m^{\prime}, m^{\prime}$. (the last $m^{\prime}, m^{\prime}$ are the multiplicities of the two tail components. (See [MM2], p. 73, Figure 3).

The space $\pi_{\mu}(\mathcal{A})$ is also globally attached to a core component in a natural way.

1.7. We present two examples of MM-quotients for certain pseudo-periodic maps of genus 2. We will again discuss them in Sections 2 and 5 via another viewpoint. For further examples of MM-quotient, see [AI1].

Example 1.7.1. Let $\mu: \Sigma_{2} \rightarrow \Sigma_{2}$ be the pseudo-periodic map of negative twist of genus 2 as follows: The decomposition (2) for $\mu$ is written as $\Sigma_{2}=\mathscr{B}_{1} \cup \mathcal{B}_{2} \cup \mathcal{A}$, where each $\mathscr{B}_{i}(i=1,2)$ is a body connected component consisting of a onepunctured torus and $\mathcal{A}$ is a non-amphidrome annulus. The valencies $(m, \lambda, \sigma, \delta)$ on $\mathcal{B}_{1}$ are $(1,4,3,3),(1,4,3,3)$ and $(1,2,1,1)$, where $(1,4,3,3)$ is attached to the boundary curve $\partial \mathscr{B}_{1}=\partial \mathcal{A}^{(1)}$ and the others are attached to the multiple points. According to [AI1], we simply say that the total valency on $\mathscr{B}_{1}$ is $\mathbf{3} / \mathbf{4}+3 / 4+1 / 2$. By the same way, the total valency on $\mathcal{B}_{2}$ is $2 / 3+2 / 3+2 / 3$. The screw number $\boldsymbol{s}(\mathcal{A})$ is $-3 / 4-2 / 3-\boldsymbol{K}(\boldsymbol{K} \geq-1)$. The minimal pseudo-period of $\mu$ is 12 . See Figure $\mathrm{G}$ in Section 2.

The MM-quotient space $F$ of $\mu$ for $\boldsymbol{K} \geq 0^{2}$ and $\boldsymbol{K}=-1$ are written as

$$
\begin{aligned}
& F=4 F^{(1)}+\sum_{k=1}^{2} \sum_{j=1}^{3}(4-j) F_{k j}^{(1)}+2 F_{3}^{(1)}+\sum_{j=1}^{K-1} F_{j}^{(0)}+3 F^{(2)} \\
& +\sum_{k=1}^{3} \sum_{j=1}^{2}(3-j) F_{k j}^{(2)}(\boldsymbol{K} \geq 0), \\
& F=4 F^{(1)}+\sum_{j=1}^{3}(4-j) F_{2 j}^{(1)}+2 F_{3}^{(1)}+3 F_{11}^{(1)}+2 F_{12 \cdot 11}^{(1 \cdot 2)}+3 F^{(2)} \\
& +\sum_{k=2}^{3} \sum_{j=1}^{2}(3-j) F_{k j}^{(2)} \quad(K=-1) .
\end{aligned}
$$

${ }^{2}$ If $\boldsymbol{K}=0$, then $F_{13}^{(1)}=F_{12}^{(2)}$ and $\sum_{j=1}^{\boldsymbol{K}}-1 F_{j}^{(0)}$ is empty. 
The configuration of $F$ is as in Figure I in Section 2. Here $F$ has two core components $F^{(1)}$ and $F^{(2)}$, four connected components of the tails $\sum_{j=1}^{3}(4-j) F_{2 j}^{(1)}, 2 F_{3}^{(1)}$, $\sum_{j=1}^{2}(3-j) F_{2 j}^{(2)}$ and $\sum_{j=1}^{2}(3-j) F_{3 j}^{(2)}$, and one connected component of the arc $\sum_{j=1}^{3}(4-j) F_{1 j}^{(1)}+\sum_{j=1}^{K-1} F_{j}^{(0)}+\sum_{j=1}^{2} j F_{1 j}^{(2)}\left(\right.$ resp. $\left.3 F_{11}^{(1)}+2 F_{12 \cdot 11}^{(1 \cdot 2)}\right)$ for $\boldsymbol{K} \geq 0$ (resp. $\boldsymbol{K}=-1)$.

Example 1.7.2. Let $\mu: \Sigma_{2} \rightarrow \Sigma_{2}$ be the pseudo-periodic map with the same decomposition $\Sigma_{2}=\mathscr{B}_{1} \cup \mathscr{B}_{2} \cup \mathcal{A}$ as in Example 1.7.1 such that the total valency of each of $\mathscr{B}_{i}(i=1,2)$ is $\mathbf{3} / \mathbf{4}+3 / 4+1 / 2$ and $\mathcal{A}$ is an amphidrome annulus with the screw number $-3 / 4-3 / 4-2 \boldsymbol{K}(\boldsymbol{K} \geq 0)$. The minimal pseudo-period is 8 . The configuration of the MM-quotient $F$ of $\mu$ is as in Figure E. Here the MM-quotient space $F$ has one core component, two connected components of the tails and one connected component of the quasi-tail.

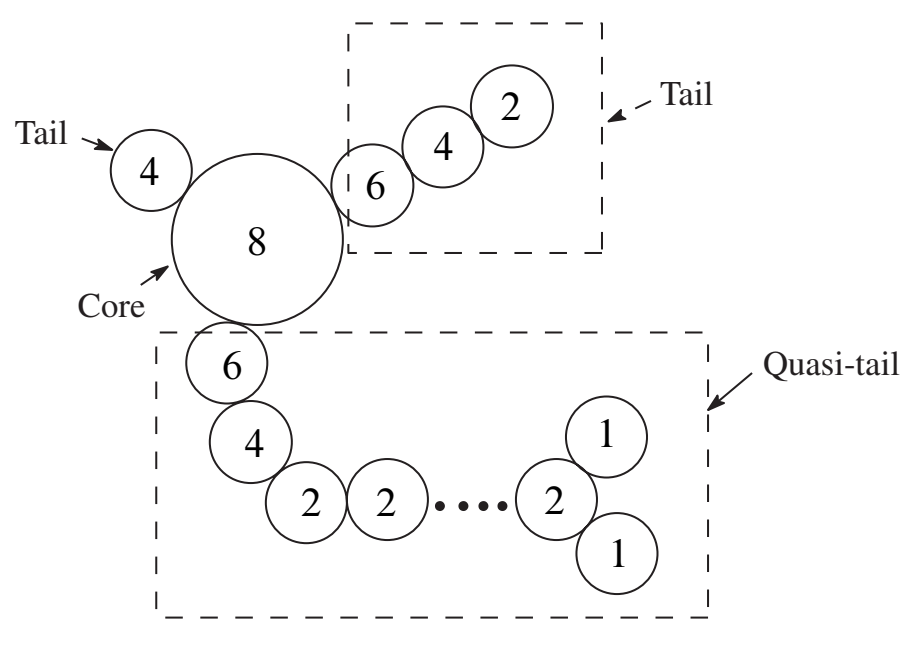

Figure E

\section{Stable reduction in biregular sense}

By a stable reduction $\tilde{f}: \widetilde{S} \rightarrow \widetilde{\Delta}$ of a degeneration $f: S \rightarrow \Delta$ of curves, we mean that $\widetilde{S}$ is birationally equivalent to the fiber product $S \times_{\Delta} \widetilde{\Delta}$ where $\widetilde{\Delta} \rightarrow \Delta$ is a cyclic cover which is totally ramified at the origin, so that the central fiber $\tilde{f}^{-1}(0)$ is a stable curve. The total space $\widetilde{S}$ may have singularities of type $A$ at the nodes of the central fiber. Note that the stable reduction is not unique for given $f$. Several proofs are known for the existence of the stable reduction in this simple situation ([DM], [AW] and $[\mathrm{BPV}]$ etc.). 
Now in this section, we present another process of the stable reduction in this situation which is different from the method of above ones. Our process seems to be precise, because we can "catch" the stable reduction explicitly in biregular sense.

2.1. Let $f: S \rightarrow \Delta$ be a proper surjective holomorphic map from a complex surface $S$ to an unit disk $\Delta=\{t \in C|| t \mid<1\}$ such that the general fiber $f^{-1}\left(t_{0}\right)\left(t_{0} \neq 0\right)$ is a nonsingular curve of genus $g \geq 1$. Let $F=f^{-1}(0)$ be the singular fiber, and let $F=\sum_{i=1}^{\gamma} \alpha_{i} F^{(i)}$ be its irreducible decomposition. Assume $f$ is normally minimal, i.e., the reduced scheme $F^{\text {red }}=\sum_{i=1}^{\gamma} F^{(i)}$ is normal crossing and any $(-1)$ component in $F_{\text {red }}$ has at least three nodes of $F^{\text {red }}$ on it. We call $f$ a normally minimal degeneration of curves of genus $g$.

Let $\mu_{f}: \Sigma_{g} \rightarrow \Sigma_{g}$ be a monodromy map of $f$, where $\Sigma_{g}$ is considered to be a fixed general fiber of $f$. The conjugacy class $\left[\mu_{f}\right] \in \widehat{\mathcal{M}}_{g}$ in the mapping class group is uniquely determined by $f$, which we call the topological monodromy of $f$. It is well known that $\left[\mu_{f}\right]$ belongs to the class of a pseudo-periodic map of negative twist ([Im], [MM1] etc.).

Let $F_{\text {ch }}=\sum_{i=1}^{\gamma} \alpha_{i} F_{\text {top }}^{(i)}$ be the numerical Chorizo space naturally obtained by the singular fiber $F=\sum_{i=1}^{\gamma} \alpha_{i} F^{(i)}$. Namely, if we only forget the analytic structure of each $F^{(i)}$, but do not forget the underlining topological structure and the multiplicity $\alpha_{i}$ and also its configuration, then we obtain $F_{\text {ch }}$.

The fundamental theorem of [MM1] says that $F_{\text {ch }}$ coincides with the MatsumotoMontesinos quotient $\Sigma_{g} /\left\langle\mu_{f}\right\rangle$ of the monodromy map $\mu_{f}$. We call $F^{(i)}$ a core (resp. tail, arc, quasi-tail) component of $F$ iff the corresponding $F_{\text {top }}^{(i)}$ is a core (resp. tail, arc, quasi-tail) component in the sense of Section 1. By changing the order if necessary, we may assume that $\left\{F^{(i)}\right\}_{i=1}^{\gamma^{\prime}}$ (for some $1 \leq \gamma^{\prime} \leq \gamma$ ) are core components and $\left\{F^{(i)}\right\}_{i=\gamma^{\prime}+1}^{\gamma}$ are non-core (i.e., tail, arc and quasi-tail) components.

Since $\left\{F^{(i)}\right\}_{i=\gamma^{\prime}+1}^{\gamma}$ is a proper subset (or empty set) of $F^{\text {red }}=\sum_{i=1}^{\gamma} F^{(i)}$, the intersection matrix of $\left\{F^{(i)}\right\}_{i=\gamma^{\prime}+1}^{\gamma}$ is negative definite. Therefore we have the bimeromorphic holomorphic map $\rho: S \rightarrow S^{\#}$ which contract $\left\{F^{(i)}\right\}_{i=\gamma^{\prime}+1}^{\gamma}$ to points by Grauert's theorem [Gr]. Let $f^{\sharp}: S^{\sharp} \rightarrow \Delta$ be the natural holomorphic map which satisfies $f^{\sharp} \circ \rho=f$. Putting $F_{i}^{\sharp}=\rho\left(F^{(i)}\right)$ for $1 \leq i \leq \gamma^{\prime}$, the fiber $F^{\sharp}:=\left(f^{\sharp}\right)^{-1}(0)$ is written as $F^{\#}=\sum_{i=1}^{\gamma^{\prime}} \alpha_{i} F_{i}^{\#}$.

Now the two-dimensional analytic space $S^{\sharp}$ has at most isolated singularities $P$ so that the support of $P$ is contained in $\left(F^{\sharp}\right)^{\text {red }}=\sum_{i=1}^{\gamma^{\prime}} F_{i}^{\#}$. Moreover one of the following is satisfied:

(i) $\left(F^{\sharp}\right)^{\text {red }}$ is smooth at $P$, and $P$ is the contraction image by $\rho$ of a tail.

(ii) $\left(F^{\sharp}\right)^{\text {red }}$ is singular at $P$, and $P$ is the contraction image by $\rho$ of an arc.

(iii) $\left(F^{\sharp}\right)^{\text {red }}$ is singular at $P$, and $P$ is the contraction image by $\rho$ of a quasi-tail. 
We determine the type of singularity at $P$ of $S^{\#}$ in the sense of Brieskorn $[\mathrm{Br}]$ for each case. First we consider (i). Let $(m, \lambda, \sigma, \delta)$ be the valency of the multiple point corresponding to the tail, and go back to the situation in Section 1.4. The point $P$ is the contraction image of the chain $\sum_{i=1}^{r} m n_{i} E_{i}$ of rational curves. By identifying this chain with the divisor on $S$ which is a part of components of $F$, we have

$$
0=F E_{i}=m\left(n_{i-1}+n_{i} E_{i}^{2}+n_{i+1}\right)
$$

for $1 \leq i \leq r$ (where $n_{r+1}$ is assumed to be 0 ). It follows that $E_{i}^{2}=\left(-n_{i-1}-\right.$ $\left.n_{i+1}\right) / n_{i}=-K_{i}$. Namely, this is the Hirzebruch-Jung string whose components have the self-intersection numbers $-K_{1}, \ldots,-K_{r}$. Therefore by the wellknown argument, the germ $\left(S^{\sharp}, P\right)$ is a cyclic quotient singularity of type $C_{\lambda, \delta}$, i.e., the germ at the origin of the quotient $C^{2} /(\mathbb{Z} / \lambda \mathbb{Z})$ by the action $\left(z_{1}, z_{2}\right) \rightarrow$ $\left(\boldsymbol{e}(1 / \lambda) z_{1}, \boldsymbol{e}(\delta / \lambda) z_{2}\right)$.

Second we consider (ii). We go back to Section 1.5, and consider the nonamphidrome annulus corresponding to the arc. The point $P$ is the contraction image of the Hirzebruch-Jung string whose self-intersection numbers of the components coincides with the self-intersection sequence of the continued linear fraction $\Phi\left(\lambda^{(1)} / \sigma^{(1)}, \lambda^{(2)} / \sigma^{(2)}, \boldsymbol{K}\right)$. Therefore it follows from the tautness of this type of singularity, Lemma 1.5.1, Lemma 1.5.3 and Theorem 1.5.4 that $\left(S^{\sharp}, P\right)$ is a cyclic quotient singularity of type $C_{d, \bar{v}}$.

We consider (iii). The point $P$ is the contraction image of the tree of rational curves whose dual graph has Dynkin diagram of type $\mathrm{D}$ as in Section 1.6. By the argument in $[\mathrm{Br}],\left(S^{\sharp}, P\right)$ is a dihedral quotient singularity of type $\langle b ; 2,1 ; 2,1 ; \delta+$ $\lambda(\boldsymbol{K}+1), \delta+\lambda \boldsymbol{K}\rangle$ in p. 347 of [Br]. For more simplified notation as in [R], we call it of type $D_{\xi+\lambda, \xi}$ by putting $\xi=\delta+\lambda \boldsymbol{K}$. Summarizing the above argument we obtain

Lemma 2.1.1. In Case (i), ( $\left.S^{\sharp}, P\right)$ is a cyclic quotient singularity of type $C_{\lambda, \delta}$.

In Case (ii), $\left(S^{\sharp}, P\right)$ is a cyclic quotient singularity of type $C_{d, \bar{v}}$, where $d, \bar{v}$ are given in (7)-(9).

In Case (iii), $\left(S^{\sharp}, P\right)$ is a dihedral quotient singularity of type $D_{\xi+\lambda, \xi}$.

2.2. In general, let $M$ be an analytic space and $M \rightarrow \Delta$ be a fibration of curves. Let $a$ be a positive integer, and $h^{(a)}: \Delta^{(a)} \rightarrow \Delta$ be the covering between small disks defined by $z \rightarrow z^{a}$. Let $M^{(a)}$ be the normalization of the analytic space $M \times{ }_{\Delta} \Delta^{(a)}$. We call the natural morphism $M^{(a)} \rightarrow \Delta^{(a)}$ the pure a-th root fibration ${ }^{3}$ of $M \rightarrow \Delta$. We set $\tilde{h}^{(a)}: M^{(a)} \rightarrow M$ the natural morphism. The following is a slightly more precise version of the classical stable reduction theorem:

\footnotetext{
${ }^{3}$ This notion is slightly different from the root fibration in [BPV] p.92, because the modification after normalization is unused.
} 
Theorem 2.2.1. (i) Let $N$ be a pseudo-period of $\mu_{f}$. Then the following hold.

(ia) The pure $N$-th root fibration $f^{(N)}: S^{(N)} \rightarrow \Delta^{(N)}$ of $f^{\sharp}: S^{\sharp} \rightarrow \Delta$ is a stable reduction of $f$. Moreover, $f^{(N)}$ is the unique stable reduction of $f$ in the birational equivalence class of $S \times{ }_{\Delta} \Delta^{(N)}$.

(ib) The cyclic group $G=\mathbb{Z} / N \mathbb{Z}$ acts holomorphically on $S^{(N)}$ so that the quotient space $S^{(N)} / G$ coincides with $S^{\#}$.

(ic) For a node $\widetilde{P}$ on the singular fiber $\widetilde{F}=\left(f^{(N)}\right)^{-1}(0)$, the germ of the point $P=\tilde{h}^{(N)}(\widetilde{P})$ on $S^{\#}$ is one of (ii) and (iii) in Lemma 2.1.2. Then the germ $(\widetilde{S}, \widetilde{P})$ is a rational double point of type $A_{n-1}(n \geq 1)$ such that the Milnor number $n$ is given by

$$
n=-\frac{N s(\mathcal{A})}{m(\mathcal{A})},
$$

where $\mathcal{A}$ is the annulus whose center curve is the vanishing cycle corresponding to $P$.

(ii) Conversely, any stable reduction of $f$ coincides with the pure $N$-th root fibration $f^{(N)}$ of $f^{\#}$ for some pseudo-period $N$ of $\mu_{f}$. In particular, $f^{\left(N_{0}\right)}$ is the minimal stable reduction in the sense that the covering degree $N_{0}$ of the base change is minimal among all of them.

Proof. Step 1. We first fix a pseudo-period $N$ of $\mu_{f}$. Let $B$ be a connected component of $\boldsymbol{B}$ in (2). Let $\left\{Q_{i}\right\}_{i=1}^{s}$ be the set of multiple points on $B$, and $\left\{\partial B_{s+1 \leq i \leq s+s^{\prime}}^{(i)}\right\}$ be the set of connected components of the boundary $\partial B$. Let $\left\{\left(m_{i}, \lambda_{i}, \sigma_{i}, \delta_{i}\right)\right\}_{1 \leq i \leq s+s^{\prime}}$ be the valency at each multiple point or boundary component. Let $N_{1}$ be the minimal period of $\mu$ at $B$, i.e., $N_{1}$ is the minimal number such that $\left(\mu_{B}\right)^{N_{1}}$ is isotopic to the identity $\operatorname{id}_{B}$. Then $N_{1}$ is a divisor of $N$ and we have $N_{1}=m_{i} \lambda_{i}$ for $1 \leq i \leq s+s^{\prime}$.

There exists a unique component of $F^{\#}$ containing $\pi_{\mu}(B)$ as a set, which we may assume to be $F_{1}^{\#}$. Let $P_{i}\left(1 \leq i \leq s+s^{\prime}\right)$ be the point on $F_{1}^{\#}$ which is the image by the contraction map $\rho$ of the tail or the arc or the quasi-tail corresponding to $Q_{i}$ or $\partial B^{(i)}$. The curve $F_{1}^{\#}$ itself is smooth or irreducible with nodes.

Now we choose an open set $U^{\sharp}$ of $S^{\sharp}$ containing the divisor $\alpha_{1} F_{1}^{\sharp}$ so that the complement of $\alpha_{1} F_{1}^{\sharp}$ in $U^{\sharp} \cap F^{\sharp}$ is empty or consists of small punctured disks with some multiplicities, and satisfies $f^{\sharp}\left(U^{\sharp}\right)=\Delta$ (see Figure F).

First we consider the pure $N_{1}$-th root fibration $f_{\text {loc }}^{\left(N_{1}\right)}: U^{\left(N_{1}\right)} \rightarrow \Delta^{\left(N_{1}\right)}$ of $f_{U^{\sharp}}^{\#}: U^{\sharp} \rightarrow \Delta$. The unique closed component $F_{1}^{\left(N_{1}\right)}$ of the central fiber $F^{\left(N_{1}\right)}=$ $\left(f_{\text {loc }}^{\left(N_{1}\right)}\right)^{-1}(0)$ is itself smooth or irreducible with nodes. Let $\hat{\tau}_{1}: \bar{F}_{1}^{\left(N_{1}\right)} \rightarrow F_{1}^{\left(N_{1}\right)}$ be the normalization, and let $\hat{h}: \bar{F}_{1}^{\left(N_{1}\right)} \rightarrow \bar{F}_{1}^{\#}$ be the lift of the restriction map

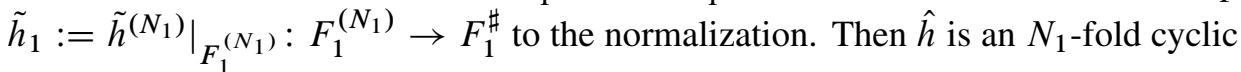
covering whose branch points coincides with $\left\{\tau_{1}^{-1}\left(P_{i}\right)\right\}_{1 \leq i \leq s+s^{\prime}}$. Moreover the total 
valency data of the periodic automorphism of $\bar{F}_{1}^{\left(N_{1}\right)}$ induced by this covering transformation coincides with $\left\{\left(m_{i}, \lambda_{i}, \sigma_{i}, \delta_{i}\right)\right\}_{1 \leq i \leq s+s^{\prime}}$. (cf. [AI1], §1.) Since the natural morphism $\tilde{h}_{\text {loc }}^{\left(N_{1}\right)}: U^{\left(N_{1}\right)} \rightarrow U^{\sharp}$ is unramified covering over $U^{\sharp} \backslash \bigsqcup_{1 \leq i \leq s+s^{\prime}} P_{i}$, the singularities of $U^{\left(N_{1}\right)}$ are at most on $\left(\tilde{h}^{\left(N_{1}\right)}\right)^{-1}\left(P_{i}\right)\left(1 \leq i \leq s+s^{\prime}\right)$.

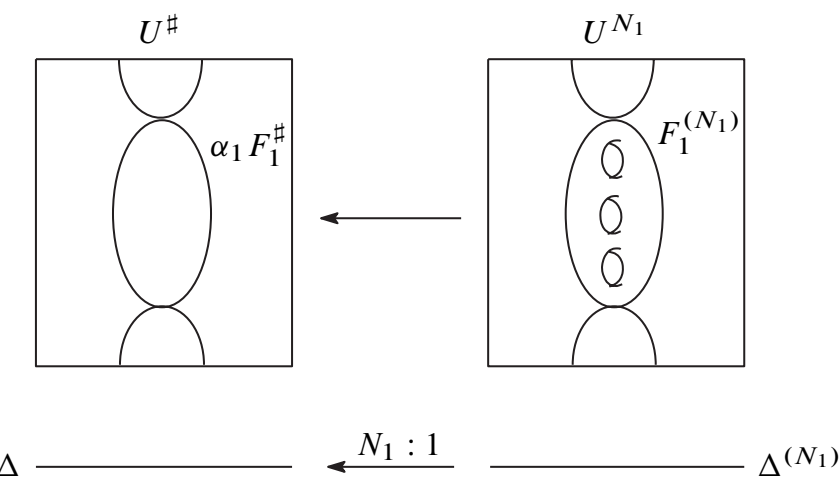

Figure F

But, over $\left(\tilde{h}_{\mathrm{loc}}^{\left(N_{1}\right)}\right)^{-1}\left(P_{i}\right)$ for $1 \leq i \leq s$, i.e., over the tail-contracting point, we claim that $U^{\left(N_{1}\right)}$ is non-singular.

Indeed, since the germ $\left(U^{\sharp}, P_{i}\right)$ is a cyclic quotient singularity of type $C_{\lambda_{i}, \delta_{i}}$, the local fundamental group $\pi_{U \sharp, P_{i}}$ is isomorphic to $\mathbb{Z} / \lambda_{i} \mathbb{Z}$ ([Br]). By looking at the local monodromy, this group is trivialized by the $\lambda_{i}$-fold cyclic cover whose Galois group is the stabilizer of each ramification point over $\left(\tilde{h}^{\left(N_{1}\right)}\right)^{-1}\left(P_{i}\right)$. Since the germ of the point with trivial local fundamental group is nothing but the germ of a smooth point ([Mu]), $\left(\tilde{h}^{\left(N_{1}\right)}\right)^{-1}\left(P_{i}\right)$ consists of $m_{i}$ smooth points.

The total space $U^{(N)}$ of the pure $N$-th root fibration $f_{\text {loc }}^{(N)}: U^{(N)} \rightarrow \Delta^{(N)}$ of $f_{U}^{\sharp}$ is also smooth on the pull back of $P_{i}(1 \leq i \leq s)$, because the natural morphism $U^{(N)} \rightarrow U^{\left(N_{1}\right)}$ clearly lifts these smooth points to smooth points of $U^{(N)}$.

Step 2. Next we consider the case $s+1 \leq i \leq s+s^{\prime}$. Assume $P:=P_{i}$ is an arc-contracting point. We choose an open set $U_{P} \subset U^{\sharp}$ of $P$ so that the fiber $\left(\left.f^{\sharp}\right|_{U_{P}}\right)^{-1}(0)$ consists of two multi-disks $n D^{(1)}+n^{\prime} D^{(2)}\left(D^{(1)} \cap D^{(2)}=P\right)$ and satisfies $f^{\sharp}\left(U_{P}\right)=\Delta$. We rewrite the valencies at the boundaries $\partial \mathcal{A}_{i}$ of the nonamphidrome annulus $\mathcal{A}_{i}$ by $\left(m, \lambda^{(k)}, \sigma^{(k)}, \delta^{(j)}\right)(k=1,2)$. Note that $m$ is a common divisor of $n$ and $n^{\prime}$, and the general fiber of $\left.f^{\sharp}\right|_{U_{P}}: U_{P} \rightarrow \Delta$ consists of $m$ disjoint annuli.

First we consider the pure $m$-th root fibration $f_{\text {loc }}^{(m)}: U^{(m)} \rightarrow \Delta^{(m)}$ of $\left.f^{\sharp}\right|_{U_{P}}$. The central fiber of $f_{\mathrm{loc}}^{(m)}$ consists of two multi-disks with the multiplicities $n / m$ and $n^{\prime} / m$, and the general fiber consists of an annulus. Moreover the monodromy of 
$f_{\text {loc }}^{(m)}$ is isotopic to the linear twist with the screw number $s\left(\mathcal{A}_{i}\right)$ ([MM1], §8), i.e., there exists a parametrization $\mathcal{A}_{i} \simeq[0,1] \times \mathbb{R} / \mathbb{Z} \ni(t, u)$ such that the monodromy action of $f_{\text {loc }}^{(m)}$ is written as $(t, u) \mapsto\left(t, u-\delta^{(1)} / \lambda^{(1)}+s\left(\mathscr{A}_{i}\right) t\right)$.

Next we consider the pure $N$-th root fibration $f_{\text {loc }}^{(N)}: U^{(N)} \rightarrow \Delta^{(N)}$ of $\left.f^{\sharp}\right|_{U_{P}}$. Since $N / m$ is a multiple of both of $n / m$ and $n^{\prime} / m$, the central fiber $\left(f_{\mathrm{loc}}^{(N)}\right)^{-1}(0)$ consists of two (reduced) disks $\widetilde{D}_{1}+\widetilde{D}_{2}$ so that $\widetilde{D}_{1}$ and $\widetilde{D}_{2}$ meets transversally at a point $\widetilde{P}$. The general fiber $\tilde{\mathcal{A}}:=\left(f_{\text {loc }}^{(N)}\right)^{-1}\left(t_{0}\right)\left(t_{0} \neq 0\right)$ is an annulus, and the monodromy map $\mu_{f_{\text {loc }}^{(N)}}: \tilde{\mathcal{A}} \rightarrow \tilde{\mathcal{A}}$ is isotopic to the linear twist with the screw number $(N / m) \boldsymbol{s}\left(\mathcal{A}_{i}\right)=(N / m)\left(-\left(\delta^{(1)} / \lambda^{(1)}\right)-\left(\delta^{(2)} / \lambda^{(2)}\right)-\boldsymbol{K}\right)$.

Since $N / m$ is a multiple of $\operatorname{lcm}\left(\lambda^{(1)}, \lambda^{(2)}\right)$, this map is an integral Dehn twist of $-(N / m) s\left(\mathcal{A}_{i}\right)$ times to right hand direction. Therefore the numerical Chorizo space $\tilde{\mathcal{A}} /\left\langle\mu_{f_{\mathrm{loc}}^{(N)}}\right\rangle$ consists of two disks and a $\boldsymbol{P}^{1}$-chain of length $-(N / m) \boldsymbol{s}\left(\mathcal{A}_{i}\right)-1$ whose multiplicities of all the components are one, i.e., the chain of $(-2)$ curves of length $-(N / m) s\left(\mathcal{A}_{i}\right)-1$. Since $\left(f_{\text {loc }}^{(N)}\right)^{-1}(0)$ consists of two disks, all the components of this chain should be contracted to a point. Namely, the point $\widetilde{P} \in U^{(N)} \subset \widetilde{S}$ is nothing but the contraction image of this chain, and therefore the germ $(\widetilde{S}, \widetilde{P})$ is a rational double point of type $A_{-(N / m) s\left(\mathcal{A}_{i}\right)-1}$.

If $P$ is a quasi-tail contracting point, i.e., $\mathcal{A}_{i}$ is an amphidrome annulus, the similar argument also works. (We omit it.)

Hence $f^{(N)}: S^{(N)} \rightarrow \Delta^{(N)}$ is a stable family and the assertion (ic) is verified.

If a core component of the central fiber of $\left(f^{(N)}\right)$ is a $(-2)$ curve, it has at least three nodes of the fiber. This fact is a direct consequence of Harvey's theorem [Ha]. Therefore the uniqueness of the stable family with the fixed covering degree is also clear, which induce the assertion (ia).

Step 3. Let $N^{\prime}$ be any positive integer which is not a multiple of $N_{0}$, and we consider the pure $N^{\prime}$-th root fibration $f^{\left(N^{\prime}\right)}: S^{\left(N^{\prime}\right)} \rightarrow \Delta^{\left(N^{\prime}\right)}$ of $f^{\sharp}$. The monodromy map $\mu_{f\left(N^{\prime}\right)}: \Sigma_{g} \rightarrow \Sigma_{g}$ is isotopic to the power $\left(\mu_{f}\right)^{N^{\prime}}$, and the decomposition to the annulus part and the body part of $\mu_{f\left(N^{\prime}\right)}$ coincides with (2) of $\mu_{f}$.

Now the assumption of $N^{\prime}$ implies that there exists a connected component $B$ of the body part such that $\left.\mu_{f\left(N^{\prime}\right)}\right|_{B}$ is not isotopic to $\mathrm{id}_{B}$. Namely the period of $\left.\mu_{f\left(N^{\prime}\right)}\right|_{B}$ is greater than one. Therefore the numerical Chorizo space $\Sigma_{g} /\left\langle\mu_{f\left(N^{\prime}\right)}\right\rangle$ contains at least one core component with the multiplicity greater than one. If this core component is a $(-1)$ curve in the normally minimal fiber, then it intersects at least three tail components by [Ha]. This fiber germ cannot be transposed birationally to a stable fiber germ. Hence the assertions (ii) is clear.

The remaining assertion (ib) is also clear, because the natural action of $\mathbb{Z} / N \mathbb{Z}$ on the fiber product $S^{\sharp} \times_{\Delta} \Delta^{(N)}$ lifts to its normalization as a holomorphic Galois action by an easy argument. 
Example 2.2.2. Let $f: S \rightarrow \Delta$ be the degeneration whose topological monodromy coincides with $\mu$ which is given in Example 1.7.1. The numerical Chorizo space of the singular fiber $F$ of $f$ coincides with the MM-quotient described in Example 1.7.1. Note that $f$ is the degeneration which is listed as the types $\left[\mathrm{IV}^{*}-\mathrm{III}^{*}-m\right]$, $\left[\mathrm{IV}^{*}-\mathrm{III}^{*}-\alpha\right]$ in Namikawa-Ueno's table [NU].



Figure G

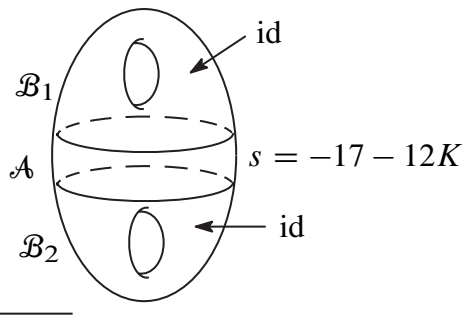

$\mu^{12}$

$12: 1$

Figure $\mathrm{H}$

Let $S \rightarrow S^{\sharp}$ be the contraction defined in Section 2.1. The irreducible decomposition of the central fiber $F^{\sharp}$ of $S^{\sharp} \rightarrow \Delta$ is written as $F^{\sharp}=4 F_{1}^{\sharp}+3 F_{2}^{\#}$. The normal analytic surface $S^{\sharp}$ has five isolated singularities $P_{1}^{(1)}, P_{2}^{(1)}, P_{12}, P_{1}^{(2)}$ and $P_{2}^{(2)}$. The points $P_{1}^{(1)}, P_{2}^{(1)}, P_{1}^{(2)}, P_{2}^{(2)}$ are tail contracting points, and are rational double points of types $A_{3}, A_{1}, A_{2}, A_{2}$ respectively. The point $P_{12}=F_{1}^{\sharp} \cap F_{2}^{\sharp}$ is an arc contraction point, and is the cyclic quotient singularity of type $C_{17+12 K}, 12+9 \mathrm{~K}$ (resp. type $C_{5,3}$ ) for $\boldsymbol{K} \geq 0$ (resp. $\boldsymbol{K}=-1$ ), because of $[2,2,3, \underbrace{2, \ldots, 2}_{\boldsymbol{K}-1}, 3,2 \rrbracket=$ $(17+12 \boldsymbol{K}) /(12+9 \boldsymbol{K})($ resp. $[2,3 \rrbracket]=5 / 3)$. See Figure $\mathbf{J}$.

Let $\tilde{f}: \tilde{S} \rightarrow \tilde{\Delta}$ be the stable family obtained by the composition of the $12: 1$ base change. The central fiber $\widetilde{F}$ consists of two components $\widetilde{F}=\widetilde{F}_{1}+\widetilde{F}_{2}$ so that $\widetilde{F}_{1}$ (resp. $\widetilde{F}_{2}$ ) is a smooth elliptic curve, since it is a 4 -fold (resp. 3 -fold) cyclic cover of $\boldsymbol{P}^{1}$ branched at 3 points whose branch indices are 4, 4, 2 (resp. 3, 3, 3). The topological monodromy around $\widetilde{F}$ coincides with $\mu^{12}$, and therefore the decomposition to the bodies and the annuli is the same as $\mu$ and the total valencies at $\mathcal{B}_{i}(i=1,2)$ are trivial and the screw number at $\mathcal{A}$ is $-17-12 K$, i.e., it is the right-handed Dehn twist of $17+12 K$ times. See Figure $\mathrm{H}_{\tilde{\widetilde{F}}}$ The surface $\widetilde{S}$ has a rational double point of type $A_{16+12 K}$ at the node $P=\widetilde{F}_{1} \cap \widetilde{F}_{2}$ as in Figure K. 


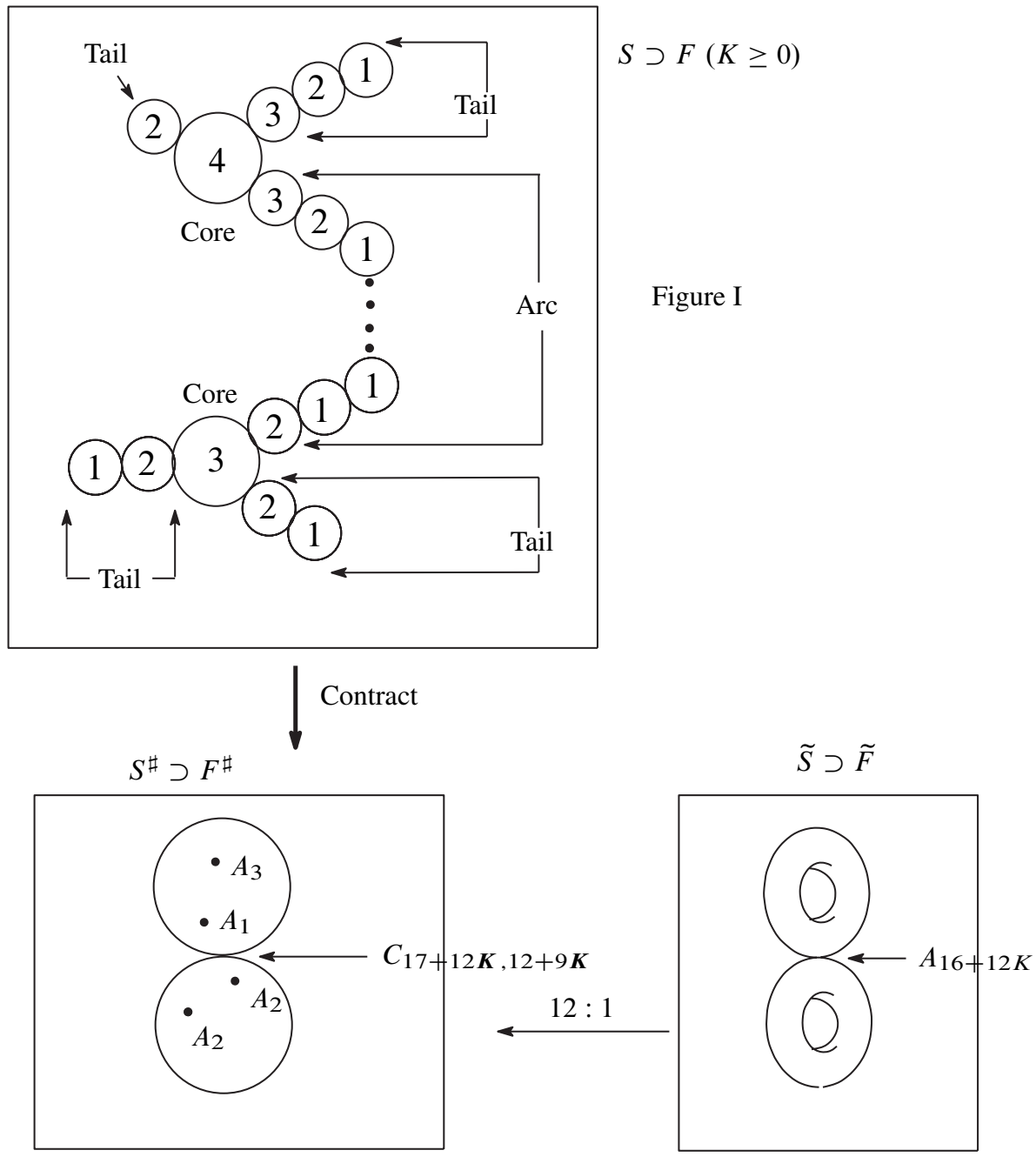

Figure $\mathbf{J}$

Figure K

Example 2.2.3. Let $f: S \rightarrow \Delta$ be the degeneration whose topological monodromy coincides with $\mu$ given in Example 1.7.2. The numerical Chorizo space of the singular fiber $F$ of $f$ coincides with the MM-quotient described in Example 1.7.2. Note that $f$ is listed as the type [2III* $-m]$ in [NU].

After the contraction $S \rightarrow S^{\sharp}$, the surface $S^{\sharp}$ has three isolated singularities on its central fiber $F^{\sharp}$ of $S^{\sharp} \rightarrow \Delta$. Two of them are rational double points of types $A_{3}$ and $A_{1}$ which are the tail contracting points, and one of them is a dihedral singularity of type $D_{7+4 K, 3+4 K}$ which is the quasi-tail contracting point. 
Let $\tilde{f}: \widetilde{S} \rightarrow \widetilde{\Delta}$ be the stable family obtained by $8: 1$ base change. The central fiber $\widetilde{F}$ consists of two smooth elliptic curves with one node so that $\widetilde{S}$ has a rational double point of type $A_{5+8 K}$ at the node.

\section{Group action and monodromy}

The notation is the same as Section 2. We fix a pseudo-period $N$ of $\mu_{f}$, and a generator $g_{0}$ of $G=\mathbb{Z} / N \mathbb{Z}$ through this section. We consider the stable reduction $\tilde{f}=f^{(N)}: \tilde{S}=S^{(N)} \rightarrow \widetilde{\Delta}=\Delta^{(N)}$ of $f: S \rightarrow \Delta$. In this section, we describe the direct relation of the action of $G$ on $\widetilde{S}$ and the monodromy data of $f$. We also review Takamura's argument [Tak] for later use.

3.1. (i) We call a smooth point $P$ on $\widetilde{F}=\tilde{f}^{-1}(0)$ an inner multiple point of $\widetilde{F}$ iff the stabilizer $\operatorname{Stab}_{G}(P)$ of $P$ in $G$ is strictly larger than the ${\operatorname{stabilizer~} \operatorname{Stab}_{G}(\widetilde{F}(i)})$ of the irreducible component $\widetilde{F}^{(i)}$ of $\widetilde{F}$ which contains $P$.

For an inner multiple point $P$, there exists a positive integer $\tilde{m}$ such that neighborhoods in $\widetilde{S}$ of the $\widetilde{m}$ mutually distinct points $P, g_{0}(P), \ldots, g_{0}^{\widetilde{m}-1}(P)$ are permuted cyclically and isomorphically to each other by the action of $g_{0}$, and $g_{0}^{\widetilde{m}}$ stabilizes the neighborhood $U_{P}$ of $P$ as a set. We choose local coordinates $(x, t)$ on $U_{P}$ so that $t$ is a lift of a parameter on $\widetilde{\Delta}$ and $x$ is a local parameter of $\widetilde{F}^{(i)}$ which satisfy $P=\{(x, t)=(0,0)\}$. Since the action of $g_{0}^{\tilde{m}}$ is locally linearizable and of finite order, there exist relatively prime natural numbers $(\tilde{\lambda}, \tilde{\delta})$ with $1 \leq \tilde{\delta} \leq \tilde{\lambda}-1$ so that the action of $g_{0}^{\widetilde{m}}$ on $U_{P}$ is written as

$$
(x, t) \longmapsto\left(e\left(\frac{\tilde{\delta}}{\tilde{\lambda}}\right) x, \boldsymbol{e}\left(\frac{1}{\tilde{\lambda} \ell}\right) t\right)
$$

where $\ell=N /(\tilde{\lambda} \tilde{m})$ is an integer, and $\boldsymbol{e}(x)=\exp (2 \pi i x)$. We also put the integer $\tilde{\sigma}$ which satisfies $\tilde{\sigma} \tilde{\delta} \equiv 1(\bmod \tilde{\lambda})$ and $1 \leq \tilde{\sigma} \leq \tilde{\lambda}-1$, and call $(\tilde{m}, \tilde{\lambda}, \tilde{\sigma}, \tilde{\delta})$ the $G$-valency at $P$.

(ii) Next assume $P$ is a node of $\widetilde{F}$. There exist disks $\mathscr{D}^{(1)}, \mathscr{D}^{(2)}$ of both sides of local irreducible components of $\widetilde{F}$ at $P$ such that $P=\mathscr{D}^{(1)} \cap D^{(2)}$. We choose a suitable local coordinate neighborhood $U_{P}$ on $\widetilde{S}$ at $P$ as

$$
U_{P}=\left\{(x, y, t) \in C^{3}\left|x y=t^{n},\right| x|\leq \epsilon,| y \mid \leq \epsilon\right\}
$$

where $\widetilde{F}=\{t=0\}, D^{(1)}=\{x=t=0\}, \mathscr{D}^{(2)}=\{y=t=0\}$ and $n$ is a positive integer. There exists a positive integer $\tilde{m}$ such that the neighborhoods in $\widetilde{S}$ of the points $P, g_{0}(P), \ldots, g_{0}^{\tilde{m}-1}(P)$ are permuted isomorphically to each other by the action of $g_{0}$ and $g_{0}^{\tilde{m}}$ stabilizes $U_{P}$ as a set without changing the boundaries, i.e., 
$g_{0}^{\tilde{m}}\left(\mathscr{D}^{(j)}\right)=\mathscr{D}^{(j)}$ for $j=1,2$. The integer $\tilde{m}$ is assumed to be the smallest among the positive integers which enjoy the above property.

Moreover, if $\widetilde{m}$ is even and $g_{0}^{\tilde{m} / 2}$ stabilizes $U_{P}$ exchanging $\mathscr{D}^{(1)}$ and $\mathscr{D}^{(2)}$, we call $P$ an amphidrome node. Otherwise, we call $P$ a non-amphidrome node.

(iia) Assume $P$ is a non-amphidrome node. Then, for $j=1,2$, there exist relatively prime natural numbers $\left(\tilde{\lambda}^{(j)}, \tilde{\delta}^{(j)}\right)$ with $1 \leq \tilde{\delta}^{(j)} \leq \tilde{\lambda}^{(j)}-1$ so that the action of $g_{0}^{\widetilde{m}}$ on $U_{P}$ is written as

$$
(x, y, t) \longmapsto\left(\boldsymbol{e}\left(\frac{\tilde{\delta}^{(1)}}{\tilde{\lambda}^{(1)}}\right) x, \boldsymbol{e}\left(\frac{\tilde{\delta}^{(2)}}{\tilde{\lambda}^{(2)}}\right) y, \boldsymbol{e}\left(\frac{1}{\operatorname{lcm}\left(\tilde{\lambda}^{(1)}, \tilde{\lambda}^{(2)}\right) \cdot \ell}\right) t\right)
$$

where $\ell=N /\left\{\operatorname{lcm}\left(\tilde{\lambda}^{(1)}, \tilde{\lambda}^{(2)}\right) \cdot \tilde{m}\right\}$ is an integer. Since the action (13) is compatible with the local equation in (12), there exists an integer $\widetilde{\boldsymbol{K}} \geq-1$ such that

$$
n=\frac{\ell}{\operatorname{gcd}\left(\tilde{\lambda}^{(1)}, \tilde{\lambda}^{(2)}\right)}\left(\tilde{\delta}^{(1)} \tilde{\lambda}^{(2)}+\tilde{\delta}^{(2)} \tilde{\lambda}^{(1)}+\tilde{\boldsymbol{K}} \tilde{\lambda}^{(1)} \tilde{\lambda}^{(2)}\right) .
$$

We also put the integer $\tilde{\sigma}^{(j)}$ which satisfies $\tilde{\sigma}^{(j)} \tilde{\delta}^{(j)} \equiv 1\left(\bmod \tilde{\lambda}^{(j)}\right)$ and $1 \leq \tilde{\sigma}^{(j)} \leq$ $\tilde{\lambda}^{(j)}-1$ for $j=1,2$, and call $\left(\tilde{m}, \tilde{\lambda}^{(j)}, \tilde{\sigma}^{(j)}, \tilde{\delta}^{(j)}\right)$ the $G$-valency at $P$. Moreover we set

$$
\tilde{\boldsymbol{s}}=-\frac{\tilde{\delta}^{(1)}}{\tilde{\lambda}^{(1)}}-\frac{\tilde{\delta}^{(2)}}{\tilde{\lambda}^{(2)}}-\tilde{\boldsymbol{K}},
$$

which we call the $G$-screw number at $P$.

(iib) Assume $P$ is an amphidrome node. There exist relatively prime natural numbers $(\tilde{\lambda}, \tilde{\delta})$ with $1 \leq \tilde{\delta} \leq \tilde{\lambda}-1$ such that $g_{0}^{\tilde{m} / 2}$ acts on $U_{P}$ by

$$
(x, y, t) \longmapsto\left(\boldsymbol{e}\left(\frac{\tilde{\delta}}{2 \tilde{\lambda}}\right) y, \boldsymbol{e}\left(\frac{\tilde{\delta}}{2 \tilde{\lambda}}\right) x, \boldsymbol{e}\left(\frac{1}{2 \tilde{\lambda} \ell}\right) t\right)
$$

where $\ell=N /(\tilde{\lambda} \tilde{m})$ is an integer. Moreover there exists a non-negative integer $\widetilde{\boldsymbol{K}}$ with

$$
n=2 \ell(\tilde{\delta}+\tilde{\boldsymbol{K}} \tilde{\lambda}) .
$$

We put the integer $\tilde{\sigma}$ in the same way, and call $(\tilde{m}, \tilde{\lambda}, \tilde{\sigma}, \tilde{\delta})$ the $G$-valency at $P$. We set

$$
\tilde{\boldsymbol{s}}=-\frac{2 \tilde{\delta}}{\tilde{\lambda}}-\widetilde{2 \boldsymbol{K}}
$$

which we call the $G$-screw number at $P$.

(iii) Let $\Gamma(\widetilde{F})$ be the dual graph of $\widetilde{F}$. This is the one-dimensional oriented graph so that the vertices correspond to the irreducible components of $\widetilde{F}$ and the oriented 
segments correspond to the nodes of $\widetilde{F}$ in a canonical way. The action of $G$ on $\widetilde{F}$ naturally induces an action on $\Gamma(\widetilde{F})$.

Now we claim that the data (i)-(iii) in this section are essentially identified with the data (i)-(iii) in Section 1.1. One way to obtain this result is to compare the types of singularities on $S^{\sharp}$ and the quotient singularities by the $G$-action in this section (see Takamura [Tak] and Section 3.2). Another way is to describe directly the monodromy map of the quotient family of the action as follows:

Theorem 3.1.1. (i) There exists a natural isomorphism $\boldsymbol{\Gamma}(\widetilde{F}) \rightarrow \Gamma\left(\mu_{f}\right)$ so that the action of $G$ on $\boldsymbol{\Gamma}(\widetilde{F})$ coincides with the action of $\mu_{f}$ on $\boldsymbol{\Gamma}\left(\mu_{f}\right)$. In particular, there exists a one-to-one correspondence between the set $\left\{F^{(i)}\right\}$ of irreducible components of $\widetilde{F}$ and the set $\left\{B^{(i)}\right\}$ of body components for $\mu_{f}$, between the set $\left\{P_{k}\right\}$ of nonamphidrome nodes of $\widetilde{F}$ and the set $\left\{\mathcal{A}_{k}\right\}$ of non-amphidrome annuli for $\mu_{f}$, and between the set $\left\{P_{\ell}\right\}$ of amphidrome nodes of $\widetilde{F}$ and the set $\left\{\mathcal{A}_{\ell}\right\}$ of amphidrome annuli for $\mu_{f}$, respectively.

(ii) Let $\left\{P_{j}\right\}$ be the set of inner multiple points on a irreducible component $F^{(i)}$, and let $\left\{Q_{j}\right\}$ be the set of multiple points on the corresponding body component $B^{(i)}$. Then there exists a one-to-one correspondence between $\left\{P_{j}\right\}$ and $\left\{Q_{j}\right\}$ such that the $G$-valency $\left(\tilde{m}\left(P_{j}\right), \tilde{\lambda}\left(P_{j}\right), \tilde{\sigma}\left(P_{j}\right), \tilde{\delta}\left(P_{j}\right)\right)$ coincides with the valency $\left(m\left(Q_{j}\right), \lambda\left(Q_{j}\right), \sigma\left(Q_{j}\right), \delta\left(Q_{j}\right)\right)$.

(iii) The G-valency $\left(\tilde{m}\left(P_{k}\right), \tilde{\lambda}^{(j)}\left(P_{k}\right), \tilde{\sigma}^{(j)}\left(P_{k}\right), \tilde{\delta}^{(j)}\left(P_{k}\right)\right)(j=1,2)$ at a nonamphidrome node $P_{k}$ coincides with the valency $\left(m\left(\mathcal{A}_{k}\right), \lambda^{(j)}\left(\mathcal{A}_{k}\right), \sigma^{(j)}\left(\mathcal{A}_{k}\right)\right.$, $\left.\delta^{(j)}\left(\mathcal{A}_{k}\right)\right)$ at the corresponding non-amphidrome annulus $\mathcal{A}_{k}$. Moreover the $G$ screw number $\tilde{\boldsymbol{s}}\left(P_{k}\right)$ coincides with the screw number $\boldsymbol{s}\left(\mathcal{A}_{k}\right)$.

(iv) The $G$-valency $\left(\tilde{m}\left(P_{\ell}\right), \tilde{\lambda}\left(P_{\ell}\right), \tilde{\sigma}\left(P_{\ell}\right), \tilde{\delta}\left(P_{\ell}\right)\right)$ at an amphidrome node $P_{\ell}$ coincides with the valency $\left(m\left(\mathcal{A}_{\ell}\right), \lambda\left(\mathcal{A}_{\ell}\right), \sigma\left(\mathcal{A}_{\ell}\right), \delta\left(\mathcal{A}_{\ell}\right)\right)$ at the corresponding amphidrome annulus $\mathcal{A}_{\ell}$. Moreover the $G$-screw number $\tilde{\boldsymbol{s}}\left(P_{\ell}\right)$ coincides with the screw number $s\left(\mathcal{A}_{\ell}\right)$.

Proof. Step 1. First, according to the well-known argument ([C], [MM1] etc.), we describe the monodromy map of $\tilde{f}$.

We consider a general fiber $\widetilde{F}_{t_{0}}=\tilde{f}^{-1}\left(t_{0}\right)\left(t_{0} \neq 0\right)$ around the stable fiber $\widetilde{F}$. For each node $P$ and its neighborhood $U_{P}$ by (12), we put $\mathcal{A}_{P, t_{0}}:=U_{P} \cap \widetilde{F}_{t_{0}}$. We have the decomposition

$$
\tilde{F}_{t_{0}}=\left(\coprod_{P} \mathcal{A}_{P, t_{0}}\right) \cup\left(\coprod_{i} \mathcal{B}_{t_{0}}^{(i)}\right)
$$

where $\bigsqcup_{i} \mathcal{B}_{t_{0}}^{(i)}$ is the decomposition to connected components of $\widetilde{F}_{t_{0}} \backslash \bigsqcup_{P} \mathcal{A}_{P, t_{0}}$. Clearly there exists a natural one-to-one correspondence between the set $\left\{\mathscr{B}_{t_{0}}^{(i)}\right\}$ and the set $\left\{\widetilde{F}^{(i)}\right\}$ of irreducible components of $\widetilde{F}$. 
Now we specially consider the fiber $\widetilde{F}_{\delta}$ for a sufficiently small positive real number $\delta$. Set $\epsilon^{\prime}=\delta^{n} / \epsilon$. We define a homeomorphism $\varphi_{P, \delta}:[0,1] \times S^{1} \rightarrow \mathcal{A}_{P, \delta}$ as follows; We first define a map $\boldsymbol{u}:[0, \epsilon] \times[0, \epsilon] \rightarrow[0,1]$ by

$$
\boldsymbol{u}(|x|,|y|)= \begin{cases}\frac{\epsilon-|y|}{2(\epsilon-|x|)} & \text { if }|x| \leq|y| \leq \epsilon, \\ 1-\frac{\epsilon-|x|}{2(\epsilon-|y|)} & \text { if }|y| \leq|x| \leq \epsilon .\end{cases}
$$

For each $u \in[0,1]$, there uniquely exists a pair of real numbers $(r(u), s(u))$ such that $r(u) s(u)=\delta^{n}, 0 \leq r(u) \leq \epsilon, 0 \leq s(u) \leq \epsilon$ and $\boldsymbol{u}(r(u), s(u))=u$. Then $(r(u), s(u))$ defines a curve connecting the point $(r(0), s(0))=\left(\epsilon^{\prime}, \epsilon\right)$ on $\partial \mathcal{A}_{P, \delta}^{(2)}=$ $\{|y|=\epsilon\}$ and the point $(r(1), s(1))=\left(\epsilon, \epsilon^{\prime}\right)$ on $\partial \mathcal{A}_{P, \delta}^{(1)}=\{|x|=\epsilon\}$. Then we define $\varphi_{P, \delta}:[0,1] \times S^{1} \rightarrow \mathcal{A}_{P, \delta}$ by

$$
\varphi_{P, \delta}(u, \alpha):=(\boldsymbol{e}(\alpha) r(u), \boldsymbol{e}(-\alpha) s(u), \delta)
$$

where $\alpha \in \mathbb{R} / \mathbb{Z} \simeq S^{1}$.

Now we define a homeomorphism $h_{\theta}: \widetilde{F}_{\delta} \rightarrow \widetilde{F}_{\boldsymbol{e}(\theta) \delta}$ for $0 \leq \theta \leq 1$ as follows; For an annulus part $\mathcal{A}_{P, \delta}$, by using the parametrization $\varphi_{P, \delta}$, we define $\left.h_{\theta}\right|_{\mathcal{A}_{P, \delta}}: \mathcal{A}_{P, \delta} \rightarrow$ $\mathcal{A}_{P, \boldsymbol{e}(\theta) \delta}$ by

$$
(x, y, \delta) \longrightarrow(\boldsymbol{e}((1-\boldsymbol{u}(|x|,|y|)) \theta n) x, \boldsymbol{e}(\boldsymbol{u}(|x|,|y|) \theta n) y, \boldsymbol{e}(\theta) \delta) .
$$

For a body part $\mathcal{B}_{\delta}^{(i)}$ which is locally defined by $t=\delta$ in a natural coordinate $(z, t)$ of $\widetilde{S}$, we define $\left.h_{\theta}\right|_{\mathcal{B}_{\delta}^{(i)}}: \mathcal{B}_{\delta}^{(i)} \rightarrow \mathcal{B}_{\boldsymbol{e}(\theta) \delta}^{(i)}$ by $(z, \delta) \rightarrow(\boldsymbol{e}(\theta n) z, \boldsymbol{e}(\theta) \delta)$. These are globally well-patched and define a homeomorphism $h_{\theta}: \widetilde{F}_{\delta} \rightarrow \widetilde{F}_{\boldsymbol{e}}(\theta) \delta$.

Then the monodromy homeomorphism is nothing but $h_{1}: \widetilde{F}_{\delta} \rightarrow \widetilde{F}_{\delta}$ by putting $\theta=1$. Since

$$
\left(\left.h_{1}\right|_{\mathcal{A}_{P, \delta}} \circ \varphi_{P, \delta}\right)(u, \alpha)=\varphi_{P, \delta}(u, \alpha-n u)
$$

by (20) and (21), $\left.h_{1}\right|_{\mathcal{A}_{P, \delta}}$ is a result of $n$-full Dehn twist. Therefore $h_{1}$ induce integral Dehn twists on the annulus part and the identity map on the body part.

Step 2. We put $\delta^{*}=\delta^{N}$, and consider the smooth fiber $F_{\delta^{*}}=f^{-1}\left(\delta^{*}\right)$. Note that $g_{0}\left(\widetilde{F}_{\delta}\right)=\widetilde{F}_{\boldsymbol{e}}(1 / N) \delta$. Let $\pi: \widetilde{S} \rightarrow \widetilde{S} / G$ be the projection, and let $\pi_{1}=\left.\pi\right|_{\widetilde{F}_{\delta}}: \widetilde{F}_{\delta} \rightarrow$ $F_{\delta^{*}}$ and $\pi_{2}=\left.\pi\right|_{\widetilde{F}_{\boldsymbol{e}(1 / n(P)) \delta}}: \widetilde{F}_{\boldsymbol{e}(1 / N) \delta} \rightarrow F_{\delta} *$ be the two isomorphisms. Since $f$ coincides with $\tilde{f}_{G}: \widetilde{S} / G \rightarrow \Delta$ over the non-critical locus on $\Delta$, the homeomorphism

$$
\boldsymbol{h}:=\pi_{2} \circ h_{1 / N} \circ\left(\pi_{1}\right)^{-1}: F_{\delta^{*}} \longrightarrow F_{\delta^{*}}
$$

is nothing but the monodromy homeomorphism of $f$. 
We set $\mathcal{A}_{P^{*}, \delta^{*}}=\pi_{1}\left(\mathcal{A}_{P, \delta}\right)$ and $\mathscr{B}_{\delta^{*}}^{(i)}=\pi_{1}\left(\mathcal{B}_{\delta}^{(i)}\right)$, and consider the decomposition

$$
F_{\delta^{*}}=\left(\coprod_{P^{*}} \mathcal{A}_{P^{*}, \delta^{*}}\right) \cup\left(\coprod_{i} \mathscr{B}_{\delta^{*}}^{(i)}\right) .
$$

Since the restriction of $\boldsymbol{h}^{N}$ to $\amalg \mathcal{B}_{\delta^{*}}^{(i)}$ is the identity map and the restriction to $\mathcal{A}_{P^{*}, \delta^{*}}$ is an integral Dehn twist on itself, $\boldsymbol{h}$ is a pseudo-periodic map so that (23) coincides with the decomposition of the annular neighborhood of the admissible system of cut curves and the body parts of $\boldsymbol{h}$. Moreover the minimal pseudo-period of $\boldsymbol{h}$ is a divisor of $N$.

Since (23) is the decomposition with respect to the monodromy $\boldsymbol{h}$, the partition graph $\boldsymbol{\Gamma}\left(\mu_{f}\right)$ is isomorphic to $\boldsymbol{\Gamma}\left(\mu_{\tilde{f}}\right)$. Since the dual graph $\boldsymbol{\Gamma}(\widetilde{F})$ is isomorphic to $\boldsymbol{\Gamma}\left(\mu_{\tilde{f}}\right)$, this is isomorphic to $\boldsymbol{\Gamma}\left(\mu_{f}\right)$. These isomorphisms are canonical and clearly compatible with the $G$-action and the monodromy action. Therefore we have the assertion (i).

Now by using (20), we define a parametrization on $\mathcal{A}_{P^{*}, \delta^{*}}$ by

$$
\varphi_{P^{*}, \delta^{*}}=\pi_{1} \circ \varphi_{P, \delta}:[0,1] \times S^{1} \longrightarrow \mathcal{A}_{P^{*}, \delta^{*}} .
$$

Assume $P$ is a non-amphidrome node. It follows from (13), (14), (15), (20), (21), (22) that

$$
\begin{aligned}
\left(\left(\left.\boldsymbol{h}^{m}\right|_{\mathcal{A}_{P^{*}, \delta^{*}}}\right) \circ \varphi_{P^{*}, \delta^{*}}\right)(u, \alpha) & =\varphi_{P^{*}, \delta^{*}}\left(u, \alpha-\frac{n \tilde{m} u}{N}+\frac{\tilde{\delta}^{(1)}}{\tilde{\lambda}^{(1)}}\right) \\
& =\varphi_{P^{*}, \delta^{*}}\left(u, \alpha+\tilde{\boldsymbol{s}} u+\frac{\tilde{\delta}^{(1)}}{\lambda^{(1)}}\right) .
\end{aligned}
$$

Namely, this is the linear twist with the screw number $\tilde{\boldsymbol{s}}$ and the valency $\tilde{\delta}^{(2)} / \tilde{\lambda}^{(2)}$ at one boundary. The valency at the other boundary coincides with $\tilde{\delta}^{(1)} / \tilde{\lambda}^{(1)}$ by (15). Therefore the assertion (iii) holds.

Assume $P$ is an amphidrome node. It follows from (15), (16), (17), (20), (21), (22) that

$$
\begin{aligned}
\left(\left(\left.\boldsymbol{h}^{m}\right|_{\mathcal{A}_{P^{*}, \delta^{*}}}\right) \circ \varphi_{P^{*}, \delta^{*}}\right)(u, \alpha) & =\varphi_{P^{*}, \delta^{*}}\left(1-u,-\alpha+\frac{\tilde{m} n u}{N}+\frac{\tilde{\delta}}{2 \tilde{\lambda}}\right) \\
& =\varphi_{P^{*}, \delta^{*}}\left(1-u,-\alpha-\frac{\tilde{\boldsymbol{s}} u}{2}+\frac{\tilde{\delta}}{2 \tilde{\lambda}}\right) .
\end{aligned}
$$

We also have $\left(\left(\left.\boldsymbol{h}^{2 m}\right|_{\mathcal{A}_{P^{*}, \delta^{*}}}\right) \circ \varphi_{P^{*}, \delta^{*}}\right)(u, \alpha)=\varphi_{P^{*}, \delta^{*}}(u, \alpha+\tilde{\boldsymbol{s}} u+\tilde{\delta} / \tilde{\lambda})$. Therefore the assertion (iv) follows.

It remains to prove (ii). For the irreducible component $\widetilde{F}^{(i)}$, we put $\widetilde{F}^{(i) *}=$ $\widetilde{F}^{(i)} \backslash \bigsqcup_{P}\left(U_{P} \cap \widetilde{F}^{(i)}\right)$ where $P$ is a node of $\widetilde{F}$ on $\widetilde{F}^{(i)}$. Then there exists a canonical analytic isomorphism $\varphi_{\boldsymbol{e}(\theta) \delta}^{(i)}: \mathscr{B}_{\boldsymbol{e}(\theta) \delta}^{(i)} \rightarrow \widetilde{F}^{(i) *}$ as Riemann surfaces with boundary. 
Now let $g_{0}^{m(i)}$ be the generator of $\operatorname{Stab}_{G}\left(\widetilde{F}^{(i)}\right)$. Then the restriction map

$$
\left.h_{m(i) / N}\right|_{\mathcal{B}_{\delta}^{(i)}}: \mathscr{B}_{\delta}^{(i)} \rightarrow \mathscr{B}_{\boldsymbol{e}(m(k) / N) \delta}^{(i)}
$$

is also an analytic isomorphism so that the composition map

$$
\left.\varphi_{\boldsymbol{e}(m(i) / N) \delta}^{(i)} \circ h_{m(i) / N}\right|_{\mathcal{B}_{\delta}^{(i)}} \circ\left(\varphi_{\delta}^{(i)}\right)^{-1}: \widetilde{F}^{(i) *} \longrightarrow \widetilde{F}^{(i) *}
$$

coincides with the analytic automorphism $\left.g_{0}^{m(i)}\right|_{\widetilde{F}^{(i) *}}: \widetilde{F}^{(i) *} \rightarrow \widetilde{F}^{(i) *}$. Therefore the body part $\mathscr{B}_{\delta^{*}}^{(i)}=\pi_{1}\left(\mathcal{B}_{\delta}^{(i)}\right)$ of $F_{\delta^{*}}$ is analytically isomorphic to $\widetilde{F}^{(i) *}$ and the restriction of the power of the monodromy map $\left.\boldsymbol{h}^{m(i)}\right|_{\mathcal{B}_{\delta^{*}}^{(i)}}: \mathscr{B}_{\delta^{*}}^{(i)} \rightarrow \mathscr{B}_{\delta^{*}}^{(i)}$ is nothing but $\left.g_{0}^{m(i)}\right|_{\widetilde{F}^{(i) *}}: \widetilde{F}^{(i) *} \rightarrow \widetilde{F}^{(i) *}$ via this isomorphism. Hence the assertion (ii) is clear by (11) and the definition of the valency.

3.2. Since Theorem 3.1.1 is established, we use the simplified notations $\widetilde{m}=m, \tilde{\lambda}=$ $\lambda, \ldots$ by omitting the "tilde". For instance, we write $(x, t) \rightarrow(\boldsymbol{e}(\delta / \lambda) x, \boldsymbol{e}(1 / \lambda \ell) t)$ for (11), etc.

We already described the singularities on $S^{\#}=\tilde{S} / G$ in Theorem 2.2.1 and Lemma 2.1.2. Here, we also explain why these types of singularities appear on $S^{\sharp}$ by the quotient of the action (11), (13) and (16). The argument of this subsection is essentially due to Takamura [Tak].

(i) We consider the neighborhood $U_{P}$ of an inner multiple point $P$. Since the action on $U_{P}$ of the cyclic group $\left\langle g_{0}^{m}\right\rangle$ generated by $g_{0}^{m}$ of (11) is not small, i.e., this group has an element which has one-dimensional fixed point locus, according to the well-known argument in singularity theory, we descend it as follows; Let $U_{P} \rightarrow V_{P^{\prime}}=\{(z, u) \in \mathscr{D} \times \mathscr{D}\}(\mathscr{D}$ is a small disk) be the map defined by $(x, t) \rightarrow(z, u)=\left(x, t^{\ell}\right)$. As the descent map of (11), we define the action $\widehat{g_{0}}$ on $V_{P^{\prime}}$ by

$$
(z, u) \longmapsto\left(\boldsymbol{e}\left(\frac{\delta}{\lambda}\right) z, \boldsymbol{e}\left(\frac{1}{\lambda}\right) u\right) .
$$

The action on $V_{P^{\prime}}$ of the cyclic group $\left\langle\widehat{g_{0}}\right\rangle \simeq \mathbb{Z} / \lambda \mathbb{Z}$ is small so that the quotient space $U_{P} /\left\langle g_{0}^{m}\right\rangle$ is isomorphic to $V_{P^{\prime}} /\left\langle\widehat{g_{0}}\right\rangle$, whose singularity at the origin is of type $C_{\lambda, \sigma}$ by (24). Namely, this is the case (i) of Lemma 2.1.2.

(ii) We consider $U_{P}$ in (12) for a node $P$. Let $\widetilde{U}_{P}=\{(X, Y) \in \mathscr{D} \times \mathscr{D}\} \rightarrow U_{P}$ be the minimal local uniformization map defined by

$$
(X, Y) \longmapsto(x, y, t)=\left(X^{n}, Y^{n}, X Y\right) .
$$

(iia) Assume $P$ is a non-amphidrome node. In order to describe the lifting to $\widetilde{U}_{P}$ of the action (13), we need the following elementary number theoretic lemma whose claim essentially due to Takamura ([Tak]) : 
Lemma 3.2.1. (i) Let $\lambda^{(1)}, \delta^{(1)}, \lambda^{(2)}, \delta^{(2)}, \boldsymbol{K}$ be natural numbers with $\left(\lambda^{(1)}, \delta^{(1)}\right)=$ (1), $\left(\lambda^{(2)}, \delta^{(2)}\right)=(1)$ as ideals in the ring of integers $\mathbb{Z}$. Put $d=\lambda^{(1)} \delta^{(2)}+$ $\lambda^{(2)} \delta^{(1)}+\boldsymbol{K} \lambda^{(1)} \lambda^{(2)}$. Then there exist natural numbers $a_{1}, a_{2}$ which satisfy

$$
a_{1}+a_{2}=\boldsymbol{K},\left(\delta^{(1)}+a_{1} \lambda^{(1)}, d\right)=\left(\delta^{(2)}+a_{2} \lambda^{(2)}, d\right)=(1) .
$$

(ii) The element $\left(\delta^{(2)}+a_{2} \lambda^{(2)}\right) \cdot\left(\delta^{(1)}+a_{1} \lambda^{(1)}\right)^{-1}$ in the multiplicative group $(\mathbb{Z} / d \mathbb{Z})^{\times}$coincides with the representative $v$ of $(8)$.

Proof. Let $p_{1}, \ldots, p_{\mu}$ be the prime factors of the number $d$. For each $p_{i}(1 \leq i \leq \mu)$, we define the subset $\mathcal{M}\left(p_{i}\right)$ of $\mathbb{Z}$ by

$$
\mathcal{M}\left(p_{i}\right)=\left\{x \in \mathbb{Z} \mid\left(\delta^{(1)}+x \lambda^{(1)}, p_{i}\right)=\left(\delta^{(2)}+(\boldsymbol{K}-x) \lambda^{(2)}, p_{i}\right)=(1)\right\} .
$$

We claim that there exists $\alpha_{i} \in \mathbb{Z}$ such that the arithmetic sequence $\left\{\alpha_{i}+k p_{i}\right\}_{k \in \mathbb{Z}}$ is contained in $\mathcal{M}\left(p_{i}\right)$;

$$
\left\{\alpha_{i}+k p_{i}\right\}_{k \in \mathbb{Z}} \subset \mathcal{M}\left(p_{i}\right) .
$$

Indeed, we first assume $\left(\lambda^{(1)}, p_{i}\right)=\left(\lambda^{(2)}, p_{i}\right)=\left(p_{i}\right)$. Then the assertion (27) is clear by $\mathcal{M}\left(p_{i}\right)=\mathbb{Z}$.

Second, we assume $\left(\lambda^{(1)}, p_{i}\right)=(1),\left(\lambda^{(2)}, p_{i}\right)=\left(p_{i}\right)$. The congruence equation $\delta^{(1)}+x \lambda^{(1)} \equiv 0\left(\bmod p_{i}\right)$ has a solution, say $x \equiv \alpha_{i}^{\prime}$. (We omit (mod $\left.p_{i}\right)$ afterwards.) We choose an $\alpha_{i} \in \mathbb{Z}$ with $\alpha_{i} \not \equiv \alpha_{i}^{\prime}$. Then $\left\{\alpha_{i}+k p_{i}\right\}_{k \in \mathbb{Z}}$ is contained in $\mathcal{M}\left(p_{i}\right)$. The case where $\left(\lambda^{(1)}, p_{i}\right)=\left(p_{i}\right),\left(\lambda^{(2)}, p_{i}\right)=(1)$ is also similar.

Third, we assume $\left(\lambda^{(1)}, p_{i}\right)=\left(\lambda^{(2)}, p_{i}\right)=(1)$. Suppose $p_{i} \geq 3$. Let $\alpha_{i}^{\prime}, \beta_{i}^{\prime}$ be natural numbers with $\delta^{(1)}+\alpha_{i}^{\prime} \lambda^{(1)} \equiv 0, \delta^{(2)}+\beta_{i}^{\prime} \lambda^{(2)} \equiv 0$. The union of elements of arithmetic sequences

$$
A=\left\{\alpha_{i}^{\prime}+k p_{i}\right\}_{k \in \mathbb{Z}} \cup\left\{\boldsymbol{K}-\beta_{i}^{\prime}+k p_{i}\right\}_{k \in \mathbb{Z}}
$$

is a proper subset of $\mathbb{Z}$. We choose a number $\alpha_{i} \in \mathbb{Z}$ with $\alpha_{i} \not \equiv \alpha_{i}^{\prime}, \alpha_{i} \not \equiv \boldsymbol{K}-\beta_{i}^{\prime}$. Then $\left\{\alpha_{i}+k p_{i}\right\}_{k \in \mathbb{Z}}$ is contained in $\mathbb{Z} \backslash A$, and therefore is contained in $\mathcal{M}\left(p_{i}\right)$.

Suppose $p_{i}=2$. Since $d \equiv 0$, one of the following occurs (modulo symmetry):

(a) $\lambda^{(1)} \equiv \lambda^{(2)} \equiv \delta^{(1)} \equiv \delta^{(2)} \equiv 1, \boldsymbol{K} \equiv 0$.

(b) $\lambda^{(1)} \equiv \lambda^{(2)} \equiv \delta^{(1)} \equiv 1, \delta^{(2)} \equiv 0, \boldsymbol{K} \equiv 1$.

(c) $\lambda^{(1)} \equiv \lambda^{(2)} \equiv 1, \delta^{(1)} \equiv \delta^{(2)} \equiv 0, K \equiv 0$.

(d) $\lambda^{(1)} \equiv \lambda^{(2)} \equiv 0, \delta^{(1)} \equiv \delta^{(2)} \equiv 1(\boldsymbol{K}$ is arbitrary $)$.

If (a) or (b) occurs, then $\{2 k\}_{k \in \mathbb{Z}} \subset \mathcal{M}(2)$. If (c) occurs, then $\{2 k+1\}_{k \in \mathbb{Z}} \subset$ $\mathcal{M}(2)$. If (d) occurs, then $\mathcal{M}(2)=\mathbb{Z}$. Hence the assertion (27) holds.

By using (27) inductively, the set of intersection

$$
\mathcal{M}\left(p_{1}\right) \cap \cdots \cap \mathcal{M}\left(p_{\mu}\right)
$$


is non-empty, because it contains an arithmetic sequence $\left\{\beta+k \prod_{i=1}^{v} p_{i}\right\}_{k \in \mathbb{Z}}$ for some $\beta \in \mathbb{Z}$. We choose an element $a_{1} \in \mathcal{M}\left(p_{1}\right) \cap \cdots \cap \mathcal{M}\left(p_{v}\right)$ and set $a_{2}=\boldsymbol{K}-a_{1}$. Then we have the assertion (i).

By the definition of $v$ and the equality $\delta^{(1)} \sigma^{(1)}=\hat{\sigma}^{(1)} \lambda^{(1)}+1$, we easily have

$$
\left(\delta^{(1)}+a_{1} \lambda^{(1)}\right) v-\left(\delta^{(2)}+a_{2} \lambda^{(2)}\right)=\left(\hat{\sigma}^{(1)}+a_{1} \sigma^{(1)}\right) d .
$$

Hence the assertion (ii) also follows.

We go back to the situation in Sections 1.5 and 3.1 (iia). The number $n$ is determined by (14). By using Lemma 3.2.1, as a lift of (13) by (25), we define the action on $\widetilde{U}_{P}$ by

$$
(X, Y) \longmapsto\left(e\left(\frac{\delta^{(1)}+a_{1} \lambda^{(1)}}{\lambda^{(1)} n}\right) X, \boldsymbol{e}\left(\frac{\delta^{(2)}+a_{2} \lambda^{(2)}}{\lambda^{(2)} n}\right) Y\right) .
$$

The cyclic action generated by (28) is not small. We descend it as follows: By putting $\tilde{\lambda}^{(i)}=\lambda^{(i)} / \operatorname{gcd}\left(\lambda^{(1)}, \lambda^{(2)}\right)(i=1,2)$, let $\widetilde{U}_{P} \rightarrow V_{P^{\prime}}=\{(\bar{X}, \bar{Y}) \in \mathscr{D} \times \mathscr{D}\}$ be the map defined by $X \mapsto \bar{X}=X^{\ell \tilde{\lambda}^{(1)}}, Y \mapsto \bar{Y}=Y^{\ell \tilde{\lambda}^{(2)}}$. Then the descent map of (28) is written as

$$
\widehat{g}_{0}:(\bar{X}, \bar{Y}) \longmapsto\left(e\left(\frac{\delta^{(1)}+a_{1} \lambda^{(1)}}{d}\right) \bar{X}, \boldsymbol{e}\left(\frac{\delta^{(2)}+a_{2} \lambda^{(2)}}{d}\right) \bar{Y}\right),
$$

which generates the small action. By (26), $\xi=\boldsymbol{e}\left(\left(\delta^{(1)}+a_{1} \lambda^{(1)}\right) / d\right)$ is a primitive $d$-th root of unity and the number $e\left(\left(\delta^{(2)}+a_{2} \lambda^{(2)}\right) / d\right)$ coincides with $\xi^{v}$ by Lemma 3.2.1 (ii). The quotient space $U_{P} /\left\langle g_{0}^{m}\right\rangle$ is isomorphic to $V_{P^{\prime}} /\left\langle\widehat{g_{0}}\right\rangle$, whose singularity at the origin is of type $C_{d, v}$. Namely this is the case (ii) of Lemma 2.1.2.

(iib) Assume $P$ is an amphidrome node. The action which is the lift by (25) of the cyclic group generated by (16) is generated by two maps

$$
\begin{aligned}
& h_{0}:(X, Y) \longmapsto\left(e\left(\frac{\delta+a_{1} \lambda}{\lambda n}\right) X, \boldsymbol{e}\left(\frac{\delta+a_{2} \lambda}{\lambda n}\right) Y\right), \\
& h_{1}:(X, Y) \longmapsto\left(e\left(\frac{1}{2 \lambda \ell}\right) Y, \boldsymbol{e}\left(\frac{1}{2 \lambda \ell}\right) X\right),
\end{aligned}
$$

where $n=\ell(2 \delta+2 \lambda \boldsymbol{K})$ and $\left(a_{1}, a_{2}\right)$ is a pair of integers with $a_{1}+a_{2}=2 \boldsymbol{K},(\delta+$ $\left.a_{1} \lambda, \lambda n\right)=\left(\delta+a_{2} \lambda, \lambda n\right)=1$. Note that $h_{1}^{2}$ is contained in the subgroup generated by $h_{0}$. This action is not small. Let $\widetilde{U}_{P} \rightarrow V_{P^{\prime}}=\{(\bar{X}, \bar{Y}) \in \mathscr{D} \times \mathbb{D}\}$ be the map defined by $(X, Y) \mapsto(\bar{X}, \bar{Y})=\left(X^{\ell}, Y^{\ell}\right)$. By putting $\xi=\delta+\lambda \boldsymbol{K}$, the descent 
action is written as

$$
\begin{aligned}
& (\bar{X}, \bar{Y}) \longmapsto\left(e\left(\frac{\delta+a_{1} \lambda}{2 \lambda \xi}\right) \bar{X}, e\left(\frac{\delta+a_{2} \lambda}{2 \lambda \xi}\right) \bar{Y}\right), \\
& (\bar{X}, \bar{Y}) \longmapsto\left(e\left(\frac{1}{2 \lambda}\right) \bar{Y}, \boldsymbol{e}\left(\frac{1}{2 \lambda}\right) \bar{X}\right),
\end{aligned}
$$

which generate the small action, and the germ of singularity at the origin of the

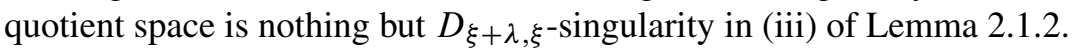

\section{Dedekind sum}

In this section, we prepare some number-theoretic arguments which relate to the Dedekind sum.

4.1. Let $(\lambda, \sigma)$ be any pair of mutually prime natural numbers with $\lambda \geq 2,1 \leq \sigma \leq$ $\lambda-1$. Let

$$
s(\sigma, \lambda):=\sum_{k=1}^{\lambda-1}\left(\left(\frac{k}{\lambda}\right)\right)\left(\left(\frac{k \sigma}{\lambda}\right)\right)
$$

be the Dedekind sum with respect to $(\lambda, \sigma)$, where $((x))=x-[x]-1 / 2$ for a noninteger $x$ and $((x))=0$ for an integer $x$. ([x] is the greatest integer not exceeding $x$.) The following formula is classical.

Theorem 4.1.1. Let $k_{1}, k_{2}$ be any integers. Then

$$
\sum_{j} \cot \frac{\pi j}{k_{1} \lambda} \cot \frac{\pi \sigma j}{k_{2} \lambda}=4 k_{1} k_{2} \cdot s(\sigma, \lambda)
$$

where $j$ moves over integers satisfying $1 \leq j \leq k_{1} k_{2} \lambda-1, j \not \equiv 0\left(\bmod k_{1} \lambda\right)$ and $j \not \equiv 0\left(\bmod k_{2} \lambda\right)$.

When $k_{1}=k_{2}=1$, it is the classical Rademacher's formula. The above formula appears in Hirzebruch-Zagier [HZ] p. 179, 180.

Let $\lambda / \sigma=\llbracket\left[K_{1}, K_{2}, \ldots, K_{r} \rrbracket\right.$ be the continued linear fraction, and set $n_{0}=$ $\lambda, n_{1}=\sigma$ and $n_{i}=K_{i-1} n_{i-1}-n_{i-2}(2 \leq i \leq r+1)$. Let $\delta$ be the integer with $\sigma \delta \equiv 1(\bmod \lambda)$ and $1 \leq \delta \leq \lambda-1$. Then $s(\sigma, \lambda)$ is expressed via these data of the continued linear fraction by Myerson-Holzapfel's formula. For the geometric meaning of this formula, see also [AI2]. 
Theorem 4.1.2 (Myerson [My], Holzapfel [Hol], p. 270).

$$
s(\sigma, \lambda)=-\frac{r}{4}+\frac{1}{12}\left(\frac{\sigma+\delta}{\lambda}+\sum_{i=1}^{r} K_{i}\right)
$$

4.2. We consider the Dedekind sum with respect to the monodromy data at a nonamphidrome annulus $\mathcal{A}$. We follow the notations in Section 1.5.

\section{Lemma 4.2.1.}

$$
\frac{v+v^{*}}{d}-\frac{\sigma^{(1)}+\delta^{(1)}}{\lambda^{(1)}}-\frac{\sigma^{(2)}+\delta^{(2)}}{\lambda^{(2)}}=-\left(\frac{\delta^{(1)}}{\lambda^{(1)}}+\frac{\delta^{(2)}}{\lambda^{(2)}}\right)-\frac{\left(\lambda^{(1)}\right)^{2}+\left(\lambda^{(2)}\right)^{2}}{d \lambda^{(1)} \lambda^{(2)}} .
$$

Proof. By (7) and $\lambda^{(i)} \hat{\sigma}^{(i)}-\sigma^{(i)} \delta^{(i)}=-1$, we have

$$
\begin{aligned}
d \lambda^{(1)} \lambda^{(2)} & \left(\frac{v+v^{*}}{d}-\frac{\sigma^{(1)}+\delta^{(1)}}{\lambda^{(1)}}-\frac{\sigma^{(2)}+\delta^{(2)}}{\lambda^{(2)}}\right) \\
=- & \lambda^{(1)} \lambda^{(2)}\left\{2 \delta^{(1)} \delta^{(2)}+\left(\lambda^{(2)} \delta^{(1)}+\lambda^{(1)} \delta^{(2)}\right) \boldsymbol{K}\right\} \\
& -\left(\lambda^{(1)}\right)^{2}\left\{1+\left(\delta^{(2)}\right)^{2}\right\}-\left(\lambda^{(2)}\right)^{2}\left\{1+\left(\delta^{(1)}\right)^{2}\right\} .
\end{aligned}
$$

On the other hand, it follows from (5) that

$$
\begin{aligned}
\left(\lambda^{(2)} \delta^{(1)}+\lambda^{(1)} \delta^{(2)}\right) \boldsymbol{K} & =\lambda^{(1)} \lambda^{(2)} \boldsymbol{K}\left(\frac{\delta^{(1)}}{\lambda^{(1)}}+\frac{\delta^{(2)}}{\lambda^{(2)}}\right) \\
& =\left(d-\lambda^{(2)} \delta^{(1)}-\lambda^{(1)} \delta^{(2)}\right)(-\boldsymbol{s}(\mathcal{A})-\boldsymbol{K}) \\
& =\left\{d-\lambda^{(1)} \lambda^{(2)}(-\boldsymbol{s}(\mathcal{A})-\boldsymbol{K})\right\}(-\boldsymbol{s}(\mathcal{A})-\boldsymbol{K}) \\
& =-(\boldsymbol{s}(\mathcal{A})+\boldsymbol{K}) d-\lambda^{(1)} \lambda^{(2)}(\boldsymbol{s}(\mathcal{A})+\boldsymbol{K})^{2} .
\end{aligned}
$$

Moreover

$$
\begin{gathered}
2 \lambda^{(1)} \lambda^{(2)} \delta^{(1)} \delta^{(2)}+\left(\lambda^{(1)}\right)^{2}\left\{1+\left(\delta^{(2)}\right)^{2}\right\}+\left(\lambda^{(2)}\right)^{2}\left\{1+\left(\delta^{(1)}\right)^{2}\right\} \\
=\left(\lambda^{(2)} \delta^{(1)}+\lambda^{(1)} \delta^{(2)}\right)^{2}+\left(\lambda^{(1)}\right)^{2}+\left(\lambda^{(2)}\right)^{2} \\
=\left(\lambda^{(1)}\right)^{2}\left(\lambda^{(2)}\right)^{2}(s(\mathcal{A})+K)^{2}+\left(\lambda^{(1)}\right)^{2}+\left(\lambda^{(2)}\right)^{2} .
\end{gathered}
$$

From (31), (32) and (33) we have the assertion.

Now we consider the case $d>1$. From the argument in Sections 1.5 and 3.2 (iia), this condition is equivalent to saying that $\mathcal{A}$ does not have the invariants $\boldsymbol{K}=-1, \omega=$ 1 and $\omega^{\prime}=0$. 
Lemma 4.2.2. Assume $d>1$. We put

$$
\epsilon^{\prime}(\mathcal{A})= \begin{cases}0 & \text { if } \boldsymbol{K} \geq 0, \\ \omega+\omega^{\prime}-r-r^{\prime}+\frac{1}{3}\left(\sum_{i=\omega+1}^{r} K_{i}+\sum_{i=\omega^{\prime}+1}^{r^{\prime}} L_{i}+2\right) & \text { if } \boldsymbol{K}=-1, \\ \omega-r-r^{\prime}+\frac{1}{3}\left(\sum_{i=\omega}^{r} K_{i}+\sum_{i=1}^{r^{\prime}} L_{i}-\alpha^{*}+1\right) & \omega \geq 1, \omega^{\prime} \geq 1, \\ & \text { if } \boldsymbol{K}=-1, \\ & \omega \geq 2, \omega^{\prime}=0,\end{cases}
$$

where $\alpha^{*}=\frac{\bar{v}-v}{d}$. (See Theorem 1.5 .4 (iv).) Then we have

$$
\begin{aligned}
& -4 s(v, d)+4 s\left(\sigma^{(1)}, \lambda^{(1)}\right)+4 s\left(\sigma^{(2)}, \lambda^{(2)}\right) \\
& =\frac{d}{3 \lambda^{(1)} \lambda^{(2)}}+\frac{\left(\lambda^{(1)}\right)^{2}+\left(\lambda^{(2)}\right)^{2}}{3 d \lambda^{(1)} \lambda^{(2)}}-1+\epsilon^{\prime}(\mathcal{A}) .
\end{aligned}
$$

Proof. It follows from Lemma 1.5.1, Lemma 1.5.3, Theorem 1.5.4 and Theorem 4.1.2 that

$$
s(v, d)=\left\{\begin{array}{cc}
-\frac{r+r^{\prime}+\boldsymbol{K}-1}{4}+\frac{1}{12}\left(\frac{v+v^{*}}{d}+\sum_{i=1}^{r} K_{i}+\sum_{i=1}^{r^{\prime}} L_{i}+2 \boldsymbol{K}\right), \boldsymbol{K} \geq 0, \\
-\frac{\omega+\omega^{\prime}-1}{4}+\frac{1}{12}\left(\frac{v+v^{*}}{d}+\sum_{i=1}^{\omega} K_{i}+\sum_{i=1}^{\omega^{\prime}} L_{i}-1\right), \quad \boldsymbol{K}=-1, \\
\omega \geq 1, \omega^{\prime} \geq 1, \\
-\frac{\omega-1}{4}+\frac{1}{12}\left(\frac{v+v^{*}}{d}+\alpha^{*}+\sum_{i=1}^{\omega-1} K_{i}\right), & \boldsymbol{K}=-1, \omega \geq 2, \omega^{\prime}=0 .
\end{array}\right.
$$

Therefore, by applying Theorem 4.1.2 again to $s\left(\sigma^{(1)}, \lambda^{(1)}\right)$ and $s\left(\sigma^{(2)}, \lambda^{(2)}\right)$, we easily have the assertion from Lemma 4.2.1.

Next we consider the case $d=1$, i.e., $\boldsymbol{K}=-1, \omega=1$ and $\omega^{\prime}=0$. In order to treat this case in the same formulation as in the case $d>1$, we define a slight (trivial) generalization of Dedekind sum $s(\sigma, \lambda)$ for $\lambda=1$. Namely, for any integer $\sigma$, we define

$$
s(\sigma, 1)=0 .
$$

The analogous result of Lemma 4.2.2 for $d=1$ is as follows:

Lemma 4.2.3. Assume $d=1$. We put

$$
\epsilon^{\prime}(\mathcal{A})=-r-r^{\prime}+\frac{1}{3}\left(\sum_{i=\omega}^{r} K_{i}+\sum_{i=1}^{r^{\prime}} L_{i}+\frac{\sigma^{(1)}}{\lambda^{(1)}}+\frac{\sigma^{(2)}}{\lambda^{(2)}}-\frac{\lambda^{(2)}}{\lambda^{(1)}}-\frac{\lambda^{(1)}}{\lambda^{(2)}}+4\right) .
$$


Then we have

$$
\begin{aligned}
& -4 s(v, 1)+4 s\left(\sigma^{(1)}, \lambda^{(1)}\right)+4 s\left(\sigma^{(2)}, \lambda^{(2)}\right) \\
& =\frac{1}{3 \lambda^{(1)} \lambda^{(2)}}+\frac{\left(\lambda^{(1)}\right)^{2}+\left(\lambda^{(2)}\right)^{2}}{3 \lambda^{(1)} \lambda^{(2)}}-1+\epsilon^{\prime}(\mathcal{A}) .
\end{aligned}
$$

Proof. From $s(v, 1)=0$ and $d=\delta^{(1)} \lambda^{(2)}+\delta^{(2)} \lambda^{(1)}-\lambda^{(1)} \lambda^{(2)}=1$, the assertion easily follows from Theorem 4.1.2.

\section{Local signature defect}

5.1. Let $0<\epsilon<1$ be a sufficiently small real number. In this section, let $f: S \rightarrow$ $\Delta$ be a normally minimal degeneration of curves of genus $g$ over a closed $\epsilon$-disk $\Delta=\{t \in \mathbf{C}|| t \mid \leq \epsilon\}$ with the central fiber $F=f^{-1}(0)$. (By a little bit abuse of the notation, one may consider $f$ the restriction over a small closed disk of the degeneration defined in Section 2.1.) We denote the restriction of $f$ to its boundary by $\partial f: \partial S \rightarrow \partial \Delta \simeq S^{1}$.

Since the restricted family over the punctured disk $f^{-1}\left(\Delta^{*}\right) \rightarrow \Delta^{*}=\Delta \backslash\{0\}$ is differentiably locally trivial, there exists a Riemannian metric $h_{S}=\left\{h_{i j}\right\}$ on $S$ such that the restriction over a tubular neighborhood of $\partial S$ is a product metric. We put $h_{\partial S}$ the restriction of $h_{S}$ to $\partial S$. The choice of $h_{S}$ is not unique, and we fix one of them. Let $\operatorname{Sign} S$ be the signature of the intersection form on $H^{2}(S, \partial S ; \mathbb{Q})$, and $\eta\left(\partial S, h_{\partial S}\right)$ be the eta-invariant of Atiyah-Patodi-Singer [APS]. Inspired by Furuta's discussion $[\mathrm{Fu}]^{4}$, we put

$$
\sigma\left(f, F ; h_{\partial S}\right)=\operatorname{Sign} S+\eta\left(\partial S, h_{\partial S}\right) .
$$

Let $\tilde{f}: \widetilde{S} \rightarrow \widetilde{\Delta}$ be the stable reduction of degree $N$ of $f$, and let $\rho: \widetilde{\Delta} \rightarrow \Delta$, $\tilde{\rho}: \widetilde{S} \rightarrow S$ be the natural maps. Let $h_{\widetilde{S}}$ be a Riemannian metric on $\widetilde{S}$ so that $h_{\widetilde{S}}$ is an extension of the natural pull back of the restricted metric of $h_{S}$ to a tubular neighborhood of $\partial S$. Since the map $\tilde{\rho}$ is umramified near the boundary, it is well defined. The metric $h_{\widetilde{S}}$ is a product metric near the boundary $\partial \widetilde{S}$, and the eta-invariant $\eta\left(\partial \widetilde{S}, h_{\partial \widetilde{S}}\right)$ is well defined. We also put $\sigma\left(\tilde{f}, \widetilde{F} ; h_{\partial \widetilde{S}}\right)=\operatorname{Sign}(\widetilde{S})+\eta\left(\partial \widetilde{S}, h_{\partial \widetilde{S}}\right)$. We define

$$
\operatorname{Lsd}\left(f, F ; h_{\partial S} ; N\right):=\sigma\left(f, F ; h_{\partial S}\right)-\frac{1}{N} \sigma\left(\tilde{f}, \widetilde{F} ; h_{\partial \tilde{S}}\right),
$$

and call it the local signature defect of $\left(f, F ; h_{\partial S}\right)$ of order $N$.

\footnotetext{
${ }^{4}$ Furuta $[\mathrm{Fu}]$ discussed this type of invariants under a more general setting of the linear connection.
} 
5.2. We summarize the notations of the monodromy data of $f$. Let $\Sigma_{g}=\boldsymbol{B} \cup \boldsymbol{A}^{\prime} \cup \boldsymbol{A}^{\prime \prime}$ be the decomposition to the set of body connected components $\boldsymbol{B}=\coprod B_{i}$, the set of non-amphidrome annuli $\boldsymbol{A}^{\prime}=\bigsqcup \mathcal{A}_{j}$ and the set of amphidrome annuli $\boldsymbol{A}^{\prime \prime}=\amalg \tilde{\mathcal{A}}_{k}$ with respect to (a representative of) the monodromy map $\mu_{f}: \Sigma_{g} \rightarrow \Sigma_{g}$. Let $\boldsymbol{B} / \sim=\amalg\left[B_{i}\right]$ be the orbit decomposition with respect to the cyclic action generated by $\mu_{f}$. Namely two components $B_{i_{1}}$ and $B_{i_{2}}$ are equivalent iff $B_{i_{2}}=\left(\mu_{f}\right)^{n}\left(B_{i_{1}}\right)$ for some integer $n$, and $\boldsymbol{B} / \sim$ is its equivalence class. We set $\boldsymbol{A}^{\prime} / \sim=\amalg\left[\mathcal{A}_{j}\right]$, $\boldsymbol{A}^{\prime \prime} / \sim=\amalg\left[\widetilde{\mathcal{A}_{k}}\right]$ similarly.

Let $\left\{m_{\alpha}, \lambda_{\alpha}^{(i)}, \sigma_{\alpha}^{(i)}, \delta_{\alpha}^{(i)}\right\}_{1 \leq \alpha \leq \varphi(i)}$ be the set of all the valencies which are attached to multiple points and boundary curves of $B_{i}$. Set

$$
\lambda_{\alpha}^{(i)} / \sigma_{\alpha}^{(i)}=\llbracket\left[K_{1}(\alpha, i), K_{2}(\alpha, i), \ldots, K_{r(\alpha, i)}(\alpha, i)\right] .
$$

Let $\left(m\left(\mathcal{A}_{j}\right), \lambda^{(1)}\left(\mathcal{A}_{j}\right), \sigma^{(1)}\left(\mathcal{A}_{j}\right), \delta^{(1)}\left(\mathcal{A}_{j}\right)\right)$ and $\left(m\left(\mathcal{A}_{j}\right), \lambda^{(2)}\left(\mathcal{A}_{j}\right), \sigma^{(2)}\left(\mathcal{A}_{j}\right)\right.$, $\left.\delta^{(2)}\left(\mathcal{A}_{j}\right)\right)$ be the valencies of both boundary curves of a non-amphidrome annulus $\mathcal{A}_{j}$, and $s\left(\mathcal{A}_{j}\right)=-\delta^{(1)}\left(\mathcal{A}_{j}\right) / \lambda^{(1)}\left(\mathcal{A}_{j}\right)-\delta^{(2)}\left(\mathcal{A}_{j}\right) / \lambda^{(2)}\left(\mathcal{A}_{j}\right)-\boldsymbol{K}\left(\mathcal{A}_{j}\right)$ be the screw number. Set $\lambda^{(1)}\left(\mathcal{A}_{j}\right) / \sigma^{(1)}\left(\mathcal{A}_{j}\right)=\llbracket\left[K_{1}^{(j)}, K_{2}^{(j)}, \ldots, K_{r(j)}^{(j)} \rrbracket\right.$ and $\lambda^{(2)}\left(\mathcal{A}_{j}\right) / \sigma^{(2)}\left(\mathcal{A}_{j}\right)=$ $\llbracket\left[L_{1}^{(j)}, L_{2}^{(j)}, \ldots, L_{r^{\prime}(j)}^{(j)} \rrbracket\right.$.

Now we define a rational number $\epsilon\left(\mathcal{A}_{j}\right)$ as follows:

If $\boldsymbol{K}\left(\mathscr{A}_{j}\right) \geq 0$, put

$$
\epsilon\left(\mathcal{A}_{j}\right)=0 .
$$

If $\boldsymbol{K}\left(\mathcal{A}_{j}\right)=-1$, put

$$
\epsilon\left(\mathcal{A}_{j}\right)= \begin{cases}\frac{1}{3}\left(\sum_{i=\omega(j)+1}^{r(j)} K_{i}^{(j)}+\sum_{i=\omega^{\prime}(j)+1}^{r^{\prime}(j)} L_{i}^{(j)}-1\right), & \omega(j) \geq 1, \omega^{\prime}(j) \geq 1, \\ \frac{1}{3}\left(\sum_{i=\omega(j)+1}^{r(j)} K_{i}^{(j)}+\sum_{i=1}^{r^{\prime}(j)} L_{i}^{(j)}-\alpha^{*}(j)-2\right), & \omega(j) \geq 2, \omega^{\prime}(j)=0, \\ \frac{1}{3}\left(\sum_{i=1}^{r(j)} K_{i}^{(j)}+\sum_{i=1}^{r^{\prime}(j)} L_{i}^{(j)}-\beta^{*}(j)-2\right), & \omega(j)=1, \omega^{\prime}(j)=0,\end{cases}
$$

where $\omega(j), \omega^{\prime}(j)$ are defined in Theorem 1.5.4, $\alpha^{*}(j)$ is defined in Lemma 4.2.2 and $\beta^{*}(j)$ is defined by

$$
\beta^{*}(j)=\frac{\lambda^{(2)}\left(\mathscr{A}_{j}\right)}{\lambda^{(1)}\left(\mathscr{A}_{j}\right)}+\frac{\lambda^{(1)}\left(\mathscr{A}_{j}\right)}{\lambda^{(2)}\left(\mathscr{A}_{j}\right)}-\sum_{i=1}^{2} \frac{\sigma^{(i)}\left(\mathcal{A}_{j}\right)}{\lambda^{(i)}\left(\mathcal{A}_{j}\right)} .
$$

Moreover we put $\ell\left(\mathcal{A}_{j}\right)=N /\left\{\operatorname{lcm}\left(\lambda^{(1)}\left(\mathcal{A}_{j}\right), \lambda^{(2)}\left(\mathcal{A}_{j}\right)\right) \cdot m\left(\mathcal{A}_{j}\right)\right\}$. 
Let $\left(m\left(\widetilde{\mathscr{A}_{k}}\right), \lambda\left(\widetilde{\mathscr{A}_{k}}\right), \sigma\left(\widetilde{\mathscr{A}_{k}}\right), \delta\left(\widetilde{\mathscr{A}_{k}}\right)\right)$ be the valency of a boundary curve of the amphidrome annulus $\widetilde{\mathscr{A}}_{k}$, and $\boldsymbol{s}\left(\widetilde{\mathscr{A}}_{k}\right)=-2 \delta\left(\widetilde{\mathscr{A}}_{k}\right) / \lambda\left(\widetilde{\mathscr{A}}_{k}\right)-2 \boldsymbol{K}\left(\widetilde{\mathscr{A}}_{k}\right)$ be the screw number. Put $\ell\left(\widetilde{\mathcal{A}_{k}}\right)=N /\left\{\lambda\left(\widetilde{\mathscr{A}_{k}}\right) m\left(\widetilde{\mathscr{A}_{k}}\right)\right\}$.

Note that the above data are independent of the choice of a representative of $\left[B_{i}\right]$, $\left[\mathcal{A}_{j}\right]$ and $\left[\tilde{\mathcal{A}}_{k}\right]$ respectively.

Now the proof of the following theorem is postponed to Section 6.

\section{Theorem 5.2.1.}

$$
\begin{aligned}
\operatorname{Lsd}\left(f, F ; h_{\partial S} ; N\right)=- & \frac{1}{3} \sum_{\left[B_{i}\right]} \sum_{\alpha=1}^{\varphi(i)}\left(\frac{\sigma_{\alpha}^{(i)}+\delta_{\alpha}^{(i)}}{\lambda_{\alpha}^{(i)}}+\sum_{j=1}^{r(\alpha, i)} K_{j}(\alpha, i)\right) \\
& +\sum_{\left[\mathcal{A}_{j}\right]}\left(\frac{\operatorname{gcd}\left(\lambda^{(1)}\left(\mathcal{A}_{j}\right), \lambda^{(2)}\left(\mathcal{A}_{j}\right)\right)}{\ell\left(\mathscr{A}_{j}\right) \lambda^{(1)}\left(\mathcal{A}_{j}\right) \lambda^{(2)}\left(\mathcal{A}_{j}\right)}-\boldsymbol{K}\left(\mathcal{A}_{j}\right)+\epsilon\left(\mathscr{A}_{j}\right)\right) \\
& +\sum_{\left[\widetilde{\mathcal{A}_{k}}\right]}\left(\frac{1}{2 \ell\left(\widetilde{\mathscr{A}_{k}}\right) \lambda\left(\widetilde{\mathscr{A}_{k}}\right)}-\boldsymbol{K}\left(\widetilde{\mathscr{A}_{k}}\right)-2\right),
\end{aligned}
$$

where the summations move over all the classes of the orbit decompositions.

Since $\operatorname{Lsd}\left(f, F ; h_{\partial S} ; N\right)$ is independent of $h_{\partial S}$, we write it by $\operatorname{Lsd}(f, F ; N)$ from now on. Moreover, if we choose $N$ minimal, then we simply write it by $\operatorname{Lsd}(f, F)$ and call it the local signature defect of the degeneration $f$, which only depends on the fiber germ at $F$.

Now let $\hat{f}: \hat{S} \rightarrow \Delta$ be the semi-stable reduction of $f$. The surface $\widehat{S}$ is obtained from $\widetilde{S}$ by the resolution $\widehat{S} \rightarrow \widetilde{S}$ of rational double points of type $A$ at the double points of $\widetilde{F}$. The boundary $\partial \widehat{S}$ of $\widehat{S}$ coincides with $\partial \widetilde{S}$, and has the natural Riemannian metric $h_{\partial \hat{S}}$. For the fiber germ $(\hat{f}, \hat{F})$, we put $\sigma\left(\hat{f}, \widehat{F} ; h_{\partial \hat{S}}\right)=\operatorname{Sign}(\hat{S})+\eta\left(\partial \hat{S}, h_{\partial \hat{S}}\right)$. Similarly we define

$$
\operatorname{Lsd}\left(f, F ; h_{\partial S} ; N\right):=\sigma\left(f, F ; h_{\partial S}\right)-\frac{1}{N} \sigma\left(\hat{f}, \widehat{F} ; h_{\partial \widehat{S}}\right)
$$

If $N$ is minimal, we put

$$
\operatorname{Lsd}(f, F):=\operatorname{Lsd}\left(f, F ; h_{\partial S} ; N\right)
$$

which also depends on the fiber germ at $F .^{5}$

\footnotetext{
${ }^{5}$ In [AY], the invariant $\mathbf{L s d}(f, F)$ is called the local signature defect instead of $\operatorname{Lsd}(f, F)$. The reason is that the result of Corollary 5.2.2 is simpler than that of Theorem 5.2.1. In this paper, we emphasize the theoretical importance.
} 


\section{Corollary 5.2.2.}

$$
\begin{aligned}
\operatorname{Lsd}\left(f, F ; h_{\partial S} ; N\right)=- & \frac{1}{3} \sum_{\left[B_{i}\right]} \sum_{\alpha=1}^{\varphi(i)}\left(\frac{\sigma_{\alpha}^{(i)}+\delta_{\alpha}^{(i)}}{\lambda_{\alpha}^{(i)}}+\sum_{j=1}^{r(\alpha, i)} K_{j}(\alpha, i)\right) \\
& +\sum_{\left[\mathcal{A}_{j}\right]}\left(\frac{\delta^{(1)}\left(\mathcal{A}_{j}\right)}{\lambda^{(1)}\left(\mathcal{A}_{j}\right)}+\frac{\delta^{(2)}\left(\mathcal{A}_{j}\right)}{\lambda^{(2)}\left(\mathcal{A}_{j}\right)}+\epsilon\left(\mathcal{A}_{j}\right)\right) \\
& +\sum_{\left[\widetilde{\mathscr{A}_{k}}\right]}\left(\frac{\delta\left(\widetilde{\mathscr{A}_{k}}\right)}{\lambda\left(\widetilde{\mathscr{A}_{k}}\right)}-2\right) .
\end{aligned}
$$

Proof. Let $n\left(\mathscr{A}_{j}\right)$ be the Milnor number of the singularity of type $A$ corresponding to the non-amphidrome annulus $\mathscr{A}_{j}$ via Theorem 3.1.1. By (14), $n\left(\mathscr{A}_{j}\right)$ coincides with

$$
\begin{aligned}
& \frac{\ell\left(\mathcal{A}_{j}\right)}{\operatorname{gcd}\left(\lambda^{(1)}\left(\mathcal{A}_{j}\right), \lambda^{(2)}\left(\mathcal{A}_{j}\right)\right)} \\
& \cdot\left(\delta^{(1)}\left(\mathscr{A}_{j}\right) \lambda^{(2)}\left(\mathcal{A}_{j}\right)+\delta^{(2)}\left(\mathscr{A}_{j}\right) \lambda^{(1)}\left(\mathcal{A}_{j}\right)+\boldsymbol{K}\left(\mathcal{A}_{j}\right) \lambda^{(1)}\left(\mathscr{A}_{j}\right) \lambda^{(2)}\left(\mathcal{A}_{j}\right)\right) .
\end{aligned}
$$

Moreover the number $N$ is written as $N=\ell\left(\mathcal{A}_{j}\right) \cdot \operatorname{lcm}\left(\lambda^{(1)}\left(\mathcal{A}_{j}\right), \lambda^{(2)}\left(\mathcal{A}_{j}\right)\right)$.

Let $n\left(\widetilde{\mathcal{A}}_{k}\right)$ be the Milnor number of the singularity of type $A$ corresponding to the amphidrome annulus $\widetilde{\mathscr{A}_{k}}$. By $(17)$, we have $n\left(\widetilde{\mathscr{A}}_{k}\right)=2 \ell\left(\widetilde{\mathscr{A}}_{k}\right)\left(\delta\left(\widetilde{\mathscr{A}_{k}}\right)\right.$ $\left.+\boldsymbol{K}\left(\widetilde{\mathscr{A}_{k}}\right) \lambda\left(\widetilde{\mathscr{A}_{k}}\right)\right)$ and $N=2 \ell\left(\widetilde{\mathscr{A}_{k}}\right) \lambda\left(\widetilde{\mathscr{A}_{k}}\right)$. Therefore we have

$$
\begin{aligned}
& \frac{1}{N}(\operatorname{Sign}(\widetilde{S})-\operatorname{Sign}(\widehat{S})) \\
& =\sum_{\left[\mathcal{A}_{j}\right]} \frac{n\left(\mathcal{A}_{j}\right)-1}{N}+\sum_{\left[\widetilde{\mathcal{A}_{k}}\right]} \frac{n\left(\widetilde{\mathscr{A}_{k}}\right)-1}{N} \\
& =\sum_{\left[\mathcal{A}_{j}\right]}\left(\frac{\delta^{(1)}\left(\mathscr{A}_{j}\right)}{\lambda^{(1)}\left(\mathscr{A}_{j}\right)}+\frac{\delta^{(2)}\left(\mathscr{A}_{j}\right)}{\lambda^{(2)}\left(\mathcal{A}_{j}\right)}+\mathbf{K}\left(\mathscr{A}_{j}\right)-\frac{1}{\ell\left(\mathscr{A}_{j}\right) \cdot \operatorname{lcm}\left(\lambda^{(1)}\left(\mathscr{A}_{j}\right), \lambda^{(2)}\left(\mathcal{A}_{j}\right)\right)}\right) \\
& +\sum_{\left[\widetilde{\mathcal{A}_{k}}\right]}\left(\frac{\delta\left(\widetilde{\mathscr{A}_{k}}\right)}{\lambda\left(\widetilde{\mathscr{A}_{k}}\right)}+\mathbf{K}\left(\widetilde{\mathscr{A}_{k}}\right)-\frac{1}{2 \ell\left(\widetilde{\mathcal{A}_{k}}\right) \lambda\left(\widetilde{\mathscr{A}_{k}}\right)}\right) .
\end{aligned}
$$

On the other hand, it follows from $\eta\left(\partial \widetilde{S}, h_{\partial \widetilde{S}}\right)=\eta\left(\partial \widehat{S}, h_{\partial \widehat{S}}\right)$ that

$$
\operatorname{Lsd}\left(f, F ; h_{\partial S} ; N\right)=\operatorname{Lsd}\left(f, F ; h_{\partial S} ; N\right)+\frac{1}{N}(\operatorname{Sign}(\tilde{S})-\operatorname{Sign}(\widehat{S})) .
$$

Hence the assertion follows from Theorem 5.2.1. 
Example 5.2.3. Let $f: S \rightarrow \Delta$ be the degeneration given in Example 2.2.2. If $\boldsymbol{K}=-1$ for the unique amphidrome annulus $\mathcal{A}$, then we have $r=3, \omega=2, r^{\prime}=2$, $\omega^{\prime}=1$ and $\epsilon:=\epsilon(\mathcal{A})=(1 / 3)(2+2-1)=1$. Therefore Theorem 5.2.1 implies that

$$
\begin{aligned}
\operatorname{Lsd}(f, F)= & \operatorname{Lsd}(f, F ; 12) \\
=- & \frac{1}{3}\left\{\left(\frac{3+3}{4}+2 \cdot 3\right) \cdot 2+\left(\frac{1+1}{2}+2\right)+\left(\frac{2+2}{3}+2 \cdot 2\right) \cdot 3\right\} \\
& +\left(\frac{1}{12}-\boldsymbol{K}+\epsilon\right) \\
= & \begin{cases}-\boldsymbol{K}-\frac{45}{4}, & \boldsymbol{K} \geq 0 \\
-\frac{37}{4}, & \boldsymbol{K}=-1 .\end{cases}
\end{aligned}
$$

Moreover

$$
\boldsymbol{L s d}(f, F)= \begin{cases}-119 / 12, & \boldsymbol{K}(\mathcal{A}) \geq 0, \\ -107 / 12, & \boldsymbol{K}(\mathcal{A})=-1 .\end{cases}
$$

Example 5.2.4. For the degeneration $f: S \rightarrow \Delta$ given in Example 2.2.3, we have

$$
\begin{aligned}
\operatorname{Lsd}(f, F) & =-\frac{1}{3}\left\{\left(\frac{3+3}{4}+2 \cdot 3\right) \cdot 2+\left(\frac{1+1}{2}+2\right)\right\}+\left(\frac{1}{2 \cdot 4}-\boldsymbol{K}-2\right) \\
& =-\frac{63}{8}-\boldsymbol{K}, \\
\operatorname{Lsd}(f, F) & =-6+\left(\frac{3}{4}-2\right)=-\frac{29}{4} .
\end{aligned}
$$

5.3. We consider the global stable reduction $\tilde{\boldsymbol{f}}: \tilde{\mathcal{S}} \rightarrow \tilde{\boldsymbol{C}}$ of $\boldsymbol{f}: S \rightarrow \mathcal{C}$ of order $N$. The description of $\tilde{\boldsymbol{f}}$ is as follows:

Let $N_{i}$ be a pseudo-period of the local monodromy map around the singular fiber $F_{i}=\boldsymbol{f}^{-1}\left(P_{i}\right)(1 \leq i \leq r)$. Let $N$ be a common multiple of all of $N_{i}$. By adding a non-critical point $P_{0}$ ("a dummy point") if necessary, we can construct a cyclic branched covering $\varphi: \tilde{C} \rightarrow \mathcal{C}$ of order $N$ whose branch points coincide with $P_{0}, P_{1}, \ldots, P_{r}$. Moreover, for $1 \leq i \leq r$, the fiber $\varphi^{-1}\left(P_{i}\right)$ consists of $N / N_{i}$ points so that the ramification indices at them are $N_{i}$. The analytic surface $\widetilde{S}$ has at most A-type singularities and is birationally equivalent to $S \times e \tilde{e}$. Explicit construction is nothing but the natural patching of $N / N_{i}$-copies of the local stable reduction of the fiber germ of $F_{i}(1 \leq i \leq r)$ discussed in Section 2. Note that $f^{-1}\left(\varphi^{-1}\left(P_{0}\right)\right)$ consists of multiple fibers whose reduced schemes are nonsingular and $\tilde{S}$ itself is smooth around the fiber. 
The local signature defect of the germ of the singular fiber is nothing but the local contribution of the difference of the global signature under the global stable reduction, i.e., we have the following lemma whose proof is also postponed to Section 6.

\section{Lemma 5.3.1.}

$$
\operatorname{Sign} S-\frac{\operatorname{Sign} \tilde{S}}{N}=\sum_{i=1}^{r} \operatorname{Lsd}\left(f_{i}, F_{i} ; N_{i}\right)
$$

where $f_{i}$ is the restricted fibration of $\tilde{\boldsymbol{f}}$ to a tubular neighborhood of $F_{i}$, and $\widetilde{F}_{i}$ is its stable fiber of the local stable reduction $\tilde{f}_{i}$ of $f_{i}$ of order $N_{i}$.

\section{Proof of main theorem}

We prove Theorem 5.2.1 and Lemma 5.3.1. Our basic tool is a complex 2-dimensional version of the orbifold signature theorem with smooth boundary. We review the notations and the result of it in Section 6.1, and then apply it to our proof in Section 6.2.

6.1. In general, let $M$ be a complex 2-dimensional $\mathrm{V}$-manifold in the sense of Satake [Sa] with a real 3-dimensional manifold boundary $\partial M$. Let $M=\bigcup U$ be a V-coordinate covering, namely, the local uniformization maps $\widetilde{U} \rightarrow U$ and the finite Galois groups $G_{U}=\operatorname{Gal}(\tilde{U} / U)$ are defined with the natural compatibility conditions, i.e., if $U_{1} \subset U_{2}$, then there exist a holomorphic open embedding $\tilde{U}_{1} \hookrightarrow \tilde{U}_{2}$ and an injective group homomorphism $G_{U_{1}} \hookrightarrow G_{U_{2}}$ which induce $\tilde{U}_{1} / G_{U_{1}}=U_{1} \hookrightarrow U_{2}=\tilde{U}_{2} / G_{U_{2}}$. Since $M$ is a rational homology manifold, the signature $\operatorname{Sign} M$ over $H^{2}(M, \partial M, \mathbb{Q})$ is well defined.

Let $\left\{h_{i j}^{U}\right\}$ be a V-Riemannian metric on $M$, i.e., each $h_{i j}^{U}$ is a $G_{U}$-invariant Riemannian metric on $\tilde{U}$ with the natural compatibility conditions. We assume $\left\{h_{i j}^{U}\right\}$ induces a product metric near $\partial M$. The V-Levi-Civita connection $\{\nabla U\}$ with respect to $\left\{h_{i j}^{U}\right\}$, and the $\mathrm{V}$-curvature matrix $\left\{R_{U}\right\}$ with respect to $\{\nabla U\}$ are defined naturally ([Sa]). The V-Pontrjagin form $\left\{p_{1}(U)\right\}$ is defined by $p_{1}(U)=(2 \pi)^{-2} \operatorname{Tr}\left(R_{U}^{2}\right)$.

For each V-coordinate $U$ and an element $g \in G_{U}$, we define the equivariant Lform $L^{g}(U)$ as follows (cf. [AS], §6): If $g=$ id, put $L^{g}(U)=(1 / 3) p_{1}(U)$, i.e., the Hirzebruch L-form of degree 1 . Assume $g \neq \mathrm{id}$, and let $\widetilde{U}^{g}$ be the $g$-fixed locus on $\widetilde{U}$, which is an isolated fixed point or a fixed complex curve.

Suppose $\tilde{U}^{g}:=P$ is an isolated fixed point. Then $g$ acts on the tangent space $T_{P}(\tilde{U})$ linearly with its eigenvalues $e^{i \alpha_{g}}$ and $e^{i \beta_{g}}\left(0<\alpha_{g}, \beta_{g}<2 \pi\right)$. Then we put $L^{g}(U)=-\cot \left(\alpha_{g} / 2\right) \cot \left(\beta_{g} / 2\right)$.

Suppose $\tilde{U}^{g}:=C$ is a fixed complex curve. Then $g$ acts on each fiber of the normal bundle $\left(N_{C / \widetilde{U}}\right)_{Q}$ of a generic point $Q \in C$ linearly with its eigenvalue $e^{i \theta_{g}}$ 
$\left(0<\theta_{g}<2 \pi\right)$. Put $L^{g}(U)=\operatorname{cosec}^{2}\left(\theta_{g} / 2\right) \cdot c_{1}\left(N_{C / \tilde{U}}\right)$, where $c_{1}\left(N_{C / \tilde{U}}\right)$ is the first Chern form of $N_{C / \widetilde{U}}$.

Then the orbifold signature theorem ([Ka]) says that

$$
\operatorname{Sign} M+\eta(\partial M)=\sum_{U} \frac{1}{\left|G_{U}\right|} \sum_{g \in G_{U}} \int_{\widetilde{U}^{g}} \rho_{U} L^{g}(U),
$$

where $\rho_{U}$ is a cut function on $U$ so that the system $\left\{\rho_{U}\right\}$ defines a partition of unity on $M$ and $\eta(\partial M)$ is the eta invariant with respect to the above V-connection. Note that (35) is the "primitive version" in some sense, and Kawasaki reformulates it by describing the correction term from the L-class as the equivariant L-class on the orbifolds which are the preimages of the V-singular locus of $M$ by the natural immersion. (See theorem and corollary in $[\mathrm{Ka}]$, p. 78, 79.)

\subsection{We prove Theorem 5.2 .1 by several steps.}

Step 1 . First we consider the surface $\widetilde{S}$, which has a simple V-manifold structure. The support of the V-singular locus (i.e., the set of points whose isotropy groups in the Galois groups are non-trivial) of $\widetilde{S}$ coincides with the set of nodes $\{P\}$ on the central fiber $\widetilde{F}$ at which $\widetilde{S}$ has $A_{n-1}$-singularities $(n \geq 1)$.

We may assume that the $\mathrm{V}$-chart $U_{P}$ containing $P$ is unique, and the local uniformization map $\tilde{U}_{P} \rightarrow U_{P}$ is given by (25). Since the generator of the Galois group $G_{U_{P}} \simeq \mathbb{Z} /(n)$ acts on $\widetilde{U}_{P}$ as $(\bar{X}, \bar{Y}) \mapsto(\boldsymbol{e}(1 / n) \bar{X}, \boldsymbol{e}((n-1) / n) \bar{Y})$, it follows from Theorem 4.1.1 that

$$
\begin{aligned}
\frac{1}{\left|G_{U_{P}}\right|} \sum_{g \in G_{U_{P}} \backslash\{\mathrm{id}\}} L^{g}\left(U_{P}\right) & =-\frac{1}{n} \sum_{j=1}^{n-1} \cot \frac{\pi j}{n} \cot \frac{\pi(n-1) j}{n} \\
& =-4 s(n-1, n)=\frac{n}{3}-1+\frac{2}{3 n} .
\end{aligned}
$$

If $P=P_{\text {noam }}$ is a non-amphidrome node, by putting $d=\delta^{(1)} \lambda^{(2)}+\delta^{(2)} \lambda^{(1)}+$ $K \lambda^{(1)} \lambda^{(2)}$ and $c=\operatorname{gcd}\left(\lambda^{(1)}, \lambda^{(2)}\right)$, we have $n=(\ell d) / c$ and $N=\left(\ell \lambda^{(1)} \lambda^{(2)}\right) / c$. If $P=P_{\text {am }}$ is an amphidrome node, we have $n=2 \ell \xi=2 \ell(\delta+\lambda \boldsymbol{K})$ and $N=2 \ell \lambda$. Therefore it follows from (35) and (36) that

$$
\begin{aligned}
& \frac{1}{N}(\operatorname{Sign} \tilde{S}+\eta(\partial \tilde{S})) \\
& =\frac{1}{3 N} \int_{\widetilde{S}} p_{1}(\tilde{S})+\sum_{P_{\text {noam }}}\left(\frac{d}{3 \lambda^{(1)} \lambda^{(2)}}-\frac{c}{\ell \lambda^{(1)} \lambda^{(2)}}+\frac{2 c^{2}}{3 \ell^{2} d \lambda^{(1)} \lambda^{(2)}}\right) \\
& \quad+\sum_{P_{\mathrm{am}}}\left(\frac{\xi}{3 \lambda}-\frac{1}{2 \ell \lambda}+\frac{1}{3 \ell \lambda n}\right)
\end{aligned}
$$


where the first summation moves over all the data of non-amphidrome nodes and the second summation moves over all the data of amphidrome nodes.

Step 2. Next we consider the surface $S^{\sharp}$ defined in Section 2. The cyclic group $G=\mathbb{Z} /(N)$ acts holomorphically on $\widetilde{S}$ so that $\widetilde{S} / G$ coincides with $S^{\sharp}$. The surface $S^{\sharp}$ has the V-manifold structure via the composition of the restriction of the global covering map $\widetilde{S} \rightarrow S^{\sharp}$ and the local uniformization map of the V-chart of $\widetilde{S}$.

First we calculate the contribution of the integral of the equivariant L-form of the isolated fixed points $P$ of the Galois groups. We may assume the V-chart $U_{P}$ containing $P$ is unique. Let $G_{U_{P}}^{\star}$ be the subset of elements $g \in G_{U_{P}}$ such that $\tilde{U}_{P}^{g}=\{P\}$, i.e., $g$ has the isolated fixed point at $P$. We set

$$
L\langle P\rangle=\frac{1}{\left|G_{U_{P}}\right|} \sum_{g \in G_{U_{P}}^{\star}} L^{g}\left(U_{P}\right) .
$$

Note that $P$ is a tail contracting point, or an arc contracting point, or a quasi-tail contracting point defined in Section 2.1.

(i) Assume $P$ is a tail contracting point. Then $\widetilde{U}_{P}$ is a neighborhood of an inner multiple point with the valency $(m, \lambda, \sigma, \delta)$ in Section 3.2 (i). The group $G_{U_{P}}$ is isomorphic to $\mathbb{Z} /(\ell \lambda)$, whose generator $g_{1}$ acts on $\widetilde{U}_{P}$ as the map (11). We put $J^{\star}=\{j \in \mathbb{Z} \mid 1 \leq j \leq \ell \lambda-1, j \not \equiv 0(\bmod \lambda)\}$. Then $G_{U_{P}}^{\star}=\left\{g_{1}^{j} \mid j \in J^{\star}\right\}$. Therefore it follows from Theorem 4.1.1 that

$$
L\langle P\rangle=-\frac{1}{\ell \lambda} \sum_{j \in J^{\star}} \cot \frac{\pi j}{\ell \lambda} \cot \frac{\pi \delta j}{\lambda}=-4 s(\delta, \lambda) .
$$

(ii) Assume $P$ is an arc contracting point. Then $\widetilde{U}_{P}$ coincides with the local uniformization space of the neighborhood of the non-amphidrome node in Section 3.2 (iia). The group $G_{U_{P}}$ is isomorphic to $\mathbb{Z} /\left(\ell^{2} \lambda^{(1)} \lambda^{(2)} d / c^{2}\right)$, whose generator $g_{1}$ acts on $\widetilde{U}_{P}$ as the map (28). We put $J^{\star}=\left\{j \in \mathbb{Z} \mid 1 \leq j \leq \ell^{2} \lambda^{(1)} \lambda^{(2)} d / c^{2}-\right.$ $\left.1, j \not \equiv 0\left(\bmod \ell \lambda^{(1)} d / c\right), j \not \equiv 0\left(\bmod \ell \lambda^{(2)} d / c\right)\right\}$. Then $G_{U_{P}}^{\star}=\left\{g_{1}^{j} \mid j \in J^{\star}\right\}$. By changing the primitive $d$-th root of unity as in the argument in Section 3.2 (iia), it follows from Theorem 4.1.1 that

$$
L\langle P\rangle=-\frac{c^{2}}{\ell^{2} \lambda^{(1)} \lambda^{(2)} d} \sum_{j \in J^{\star}} \cot \frac{\pi c j}{\ell \lambda^{(1)} d} \cot \frac{\pi c j}{\ell \lambda^{(2)} d}=-4 s(v, d) .
$$

(iii) Assume $P$ is a quasi-tail contracting point. Then $\widetilde{U}_{P}$ coincides with the local uniformization space of the neighborhood of the amphidrome node in Section 3.2 (iib). The group $G_{U_{P}}$ is isomorphic to the dihedral group of order $2 \ell \lambda n=2 \ell^{2} \lambda(2 \delta+2 \lambda \boldsymbol{K})$ whose generators $h_{0}$ and $h_{1}$ act on $\tilde{U}_{P}$ as the maps (30), and is written as $G_{U_{P}}=$ $\left\{h_{0}^{j}, h_{0}^{j} h_{1} \mid 0 \leq j \leq \ell \lambda n-1\right\}$. 
The map $h_{0}^{j}$ has an isolated fixed point at $P$ iff $j \not \equiv 0(\bmod \lambda n)$. Moreover, by putting $\sigma \delta=\lambda \hat{\sigma}+1$ and $v=\sigma \delta+\lambda \hat{\sigma}+2 \sigma \lambda \boldsymbol{K}$, the action of the subgroup generated by $h_{0}$ equivalently descends to the action generated by $(z, u) \mapsto$ $(\boldsymbol{e}(v / 2 \lambda \xi) z, \boldsymbol{e}(1 / 2 \lambda \xi) u)$.

On the other hand, since $h_{0}^{j} h_{1}$ acts as $X \mapsto \boldsymbol{e}\left(1 /(2 \ell \lambda)+\left(\delta+a_{2} \lambda\right) j /(\lambda n)\right) Y$, $Y \mapsto \boldsymbol{e}\left(1 /(2 \ell \lambda)+\left(\delta+a_{1} \lambda\right) j /(\lambda n)\right) X$, it follows that the eigenvalues of this action are $\boldsymbol{e}((1+j) /(2 \ell \lambda))$ and $\boldsymbol{e}((1+j) /(2 \ell \lambda)+1 / 2)$. The map $h_{0}^{j} h_{1}$ has an isolated fixed point at $P$ iff $j \not \equiv-1(\bmod \ell \lambda)$.

Since $\cot \frac{\pi(1+j)}{4 \ell \lambda} \cot \left(\frac{\pi(1+j)}{4 \ell \lambda}+\frac{\pi}{2}\right)=-1$, it follows from Theorem 4.1.1 that

$$
L\langle P\rangle=-\frac{1}{2 \ell \lambda n}\{4 \ell \lambda n \cdot s(v, 2 \lambda \xi)-(\ell \lambda-1)\}=-2 s(v, 2 \lambda \xi)+\frac{\ell \lambda-1}{2 \ell \lambda} .
$$

Step 3. Next we calculate the contribution of the fixed complex curves $C$ on $S^{\#}$ of the Galois groups. Namely, let $C \subset \cup U_{i}$ be a covering of V-charts in $S^{\sharp}$, and let $G_{U_{i}}^{\star}(C)$ be the point-wise stabilizer of $\left.C\right|_{U_{i}}$ in $G_{U_{i}}$. Put

$$
L\langle C\rangle=\sum_{U_{i}} \frac{1}{\left|G_{U_{i}}\right|} \sum_{g \in G_{U_{i}}^{\star}(C)} \int_{C \cap U_{i}} \rho_{U_{i}} L^{g}\left(U_{i}\right) .
$$

Note that $C$ is an irreducible component of the central fiber $F^{\#}=\sum \alpha_{j} F_{j}^{\#}$, or a hyperplane around a quasi-tail contracting point. We calculate it for each case.

(i) We consider the former case. Since the normal bundle of $F_{j}^{\#}$ in $S^{\#}$ is locally trivial on a neighborhood of a generic point of $F_{j}^{\#}$, the data in order to determine $L\left\langle F_{j}^{\sharp}\right\rangle$ are concentrated on the neighborhoods of the nodes. Let $P_{j, k}$ be a point written locally as an intersection point of two components $F_{j}^{\#}$ and $F_{k}^{\#}$, and $U_{P_{j, k}}$ be the chart containing $P_{j, k}$.

Choose the coordinate $(X, Y)$ of the uniformization space $\widetilde{U}_{P_{j, k}}$ so that $Y=0$ (resp. $X=0$ ) defines the local lift $\widetilde{F}_{j}^{\sharp}$ (resp. $\widetilde{F}_{k}^{\#}$ ) of $F_{j}^{\#}\left(\right.$ resp. $F_{k}^{\#}$ ). We take another chart $U_{i}$ with $F_{j}^{\#} \cap U_{i} \neq \emptyset, U_{i} \cap U_{P_{j, k}} \neq \emptyset$ and $U_{i} \not \supset P_{j, k}$ such that the coordinate $\left(X^{\prime}, t\right)$ of the uniformization space $\widetilde{U}_{i}$ is chosen so that $t=0$ defines the lift $\widetilde{F}_{j}^{\sharp}$, and the coordinate transformation on $\widetilde{U}_{i} \cap \widetilde{U}_{P_{j, k}}$ is given by $t=X Y, X^{\prime}=X$. Since

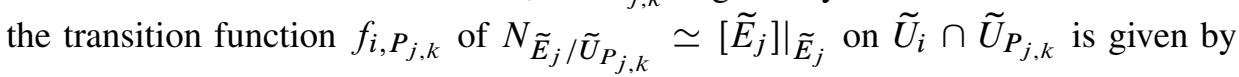
$f_{i, P_{j, k}}=Y$, the system $\left\{\left.\phi\right|_{\widetilde{U}_{P_{j, k}}}=1 / Y,\left.\phi\right|_{\widetilde{U}_{i}}=1\right\}$ defines a meromorphic section of the normal bundle of $\widetilde{E}_{j}$ over $\widetilde{U}_{i} \cup \widetilde{U}_{P_{j, k}}$. Namely, a divisor corresponding to the normal bundle has a simple pole at $\widetilde{P}_{j, k}=\{(X, Y)=(0,0)\}$. Therefore, it follows 
from the well-known local calculation (cf. $[\mathrm{GH}]$ p. 141) that

$$
\int_{\widetilde{E}_{j} \cap \widetilde{U}_{P_{j, k}}} \rho_{\widetilde{U}_{P_{j, k}}} c_{1}\left(N_{\widetilde{E}_{j} / \widetilde{U}_{P_{j, k}}}\right)=-1, \quad \int_{\widetilde{E}_{j} \cap \widetilde{U}_{i}} \rho_{\widetilde{U}_{i}} c_{1}\left(N_{\widetilde{E}_{j} / \widetilde{U}_{i}}\right)=0 .
$$

If $P_{j, k}$ is an arc contacting point, then $G_{P_{j, k}}^{\star}\left(F_{j}^{\#}\right)$ is a cyclic group of order $\ell \lambda^{(1)} / c$ generated by $n \lambda^{(2)}$-th power of the map (28). If $P_{j, k}$ is a quasi-tail contacting point, then $G_{P}^{\star}\left(F_{j}^{\sharp}\right)$ is a cyclic subgroup of order $\ell$ generated by $h_{0}^{\lambda n}$ of (30).

On the other hand, we have

$$
\sum_{k=1}^{r-1} \operatorname{cosec}^{2} \frac{\pi k}{r}=\frac{r^{2}-1}{3}
$$

for a natural number $r$ (cf. [HZ] p. 178 ). From these, we have

$$
\sum_{F_{j}^{\sharp}} L\left\langle F_{j}^{\#}\right\rangle=-\sum_{P_{\text {noam }}} \frac{c^{2}}{\ell^{2} d \lambda^{(1)} \lambda^{(2)}} \sum_{i=1}^{2} \frac{1}{3}\left\{\left(\frac{\ell \lambda^{(i)}}{c}\right)^{2}-1\right\}-\sum_{P_{\text {am }}} \frac{1}{2 \ell \lambda n} \cdot \frac{2\left(\ell^{2}-1\right)}{3} .
$$

(ii) We consider the latter case. Let $P$ be a quasi-tail contracting point, and we follow the notations in Step 2 (iii). If $j=2 k \ell \lambda-1(1 \leq k \leq n)$, the map $h_{0}^{j} h_{1}$ has a fixed curve component

$$
H_{j}:=\left\{(X, Y) \in \tilde{U}_{p} \mid \boldsymbol{e}\left(\frac{1}{2 \ell \lambda}+\frac{\left(\delta+a_{1} \lambda\right) j}{\lambda n}\right) X-Y=0\right\},
$$

so that the action of $h_{0}^{j} h_{1}$ on the normal bundle of $H_{j}$ in $\widetilde{U}_{p}$ is the reflection, i.e., with eigenvalue -1 . Since $H_{j}$ is a horizontal hyperplane with respect to the global fibration, the self-intersection $H_{j}^{2}$ on $\widetilde{U}_{p}$ (cf. [AS] p. 583) is nothing but a single point with multiplicity 1 . Therefore we have

$$
\sum_{k=1}^{n} L\left\langle H_{j}\right\rangle=\frac{1}{2 \ell \lambda n} \sum_{k=1}^{n} \operatorname{cosec}^{2} \frac{\pi}{2} \cdot H_{j}^{2}=\frac{1}{2 \ell \lambda} .
$$

Step 4. We show

$$
\begin{aligned}
& \text { Sign } S^{\sharp}+\eta\left(\partial S^{\sharp}\right) \\
& \begin{aligned}
= & \frac{1}{3} \int_{S^{\sharp}} p_{1}\left(S^{\sharp}\right)+\sum_{\left[B_{i}\right]} \sum_{\alpha=1}^{\varphi(i)}\left\{r(\alpha, i)-\frac{1}{3}\left(\frac{\sigma_{\alpha}^{(i)}+\delta_{\alpha}^{(i)}}{\lambda_{\alpha}^{(i)}}+\sum_{j=1}^{r(\alpha, i)} K_{j}(\alpha, i)\right)\right\} \\
& +\sum_{\left[\mathcal{A}_{j}\right]}\left(\frac{d}{3 \lambda^{(1)} \lambda^{(2)}}-1+\epsilon^{\prime}\left(\mathcal{A}_{j}\right)+\frac{2 c^{2}}{3 \ell^{2} d \lambda^{(1)} \lambda^{(2)}}\right)+\sum_{\left[\tilde{A}_{k}\right]}\left(\frac{\xi}{3 \lambda}+\frac{1}{3 \ell \lambda n}\right) .
\end{aligned}
\end{aligned}
$$


Indeed, it follows from (35), (38)) (39), (40), (41), (42) that

$$
\begin{aligned}
\text { Sign } S^{\sharp} & +\eta\left(\partial S^{\sharp}\right)-\frac{1}{3} \int_{S \sharp} p_{1}\left(S^{\sharp}\right) \\
= & -\sum_{\left[P_{\text {mult }}\right]} 4 s(\delta, \lambda)-\sum_{\left[P_{\text {noam }}\right]}\left\{4 s(v, d)+\frac{\left(\lambda^{(1)}\right)^{2}+\left(\lambda^{(2)}\right)^{2}}{3 d \lambda^{(1)} \lambda^{(2)}}-\frac{2 c^{2}}{3 \ell^{2} d \lambda^{(1)} \lambda^{(2)}}\right\} \\
& -\sum_{\left[P_{\mathrm{am}}\right]}\left\{2 s(v, 2 \lambda \xi)-\frac{1}{2}+\frac{\ell^{2}-1}{3 \ell \lambda}\right\},
\end{aligned}
$$

where the first, the second and the third summations move over all the monodromy data of the orbits of the monodromy map of multiple points, non-amphidrome annuli and amphidrome annuli, respectively. Since the both banks of an amphidrome annuli belong to the same orbit, we can write the right-hand side of the quality (44) as

$$
\begin{gathered}
-\sum_{[(\delta, \lambda)]} 4 s(\delta, \lambda)-\sum_{\left[P_{\text {noam }}\right]}\left\{4 s(v, d)-4 s\left(\delta^{(1)}, \lambda^{(1)}\right)-4 s\left(\delta^{(2)}, \lambda^{(2)}\right)\right. \\
\left.+\frac{\left(\lambda^{(1)}\right)^{2}+\left(\lambda^{(2)}\right)^{2}}{3 d \lambda^{(1)} \lambda^{(2)}}-\frac{2 c^{2}}{3 \ell^{2} d \lambda^{(1)} \lambda^{(2)}}\right\} \\
-\sum_{\left[P_{\mathrm{am}}\right]}\left\{2 s(v, 2 \lambda \xi)-4 s(\delta, \lambda)-\frac{1}{2}+\frac{\ell^{2}-1}{3 \ell \lambda}\right\},
\end{gathered}
$$

where the first summation moves over the valencies of the orbits of multiple points and of both sides of non-amphidrome and amphidrome annuli, i.e., the valencies of all the bodies. Moreover, for the monodromy data of an amphidrome annulus, a similar argument as in Lemma 4.2.3 shows that

$$
-2 s(v, 2 \lambda \xi)+4 s(\delta, \lambda)=\frac{\delta}{3 \lambda}+\frac{1}{6 \lambda \xi}+\frac{\boldsymbol{K}}{3}-\frac{1}{2} .
$$

Hence the assertion (43) follows from Theorem 4.1.2, Lemma 4.2.2, Lemma 4.2.3 and (45).

Step 5. Since the intersection matrix of the exceptional set is negative definite, the contraction $S \rightarrow S^{\sharp}$ induces the decrease of the dimension of the negative eigenspace of the intersection form as the same number of the exceptional curves. Therefore

$$
\operatorname{Sign} S-\operatorname{Sign} S^{\#}=-\sum_{\left[B_{i}\right]} \sum_{\alpha=1}^{\varphi(i)} r(\alpha, i)-\sum_{\left[\mathcal{A}_{j}\right]}\left(\boldsymbol{K}-1+\epsilon^{\prime \prime}\left(\mathcal{A}_{j}\right)\right)-\sum_{\left[\tilde{\mathcal{A}}_{k}\right]}(\boldsymbol{K}+2) \text {, }
$$

where

$$
\epsilon^{\prime \prime}\left(\mathcal{A}_{j}\right)= \begin{cases}0 & \text { if } \boldsymbol{K} \geq 0 \\ \omega+\omega^{\prime}-r-r^{\prime}+1 & \text { if } \boldsymbol{K}=-1\end{cases}
$$


Note that the number $\epsilon\left(\mathscr{A}_{j}\right)$ defined in Section 5.2 for a non-amphidrome annulus $\mathscr{A}_{j}$ coincides with $\epsilon^{\prime}\left(\mathcal{A}_{j}\right)-\epsilon^{\prime \prime}\left(\mathcal{A}_{j}\right)$, where $\epsilon^{\prime}\left(\mathcal{A}_{j}\right)$ is defined in Lemma 4.2.2 and Lemma 4.2.3.

By the definition of the orbifold Pontrjagin form, we have

$$
\int_{S^{\sharp}} p_{1}\left(S^{\sharp}\right)=\frac{1}{N} \int_{\widetilde{S}} p_{1}(\tilde{S}) .
$$

Moreover, since $G$ acts on $\partial \widetilde{S}$ freely, $\eta(\partial S)=\eta\left(\partial S^{\sharp}\right)$ coincides with $(1 / N) \eta(\partial \widetilde{S})$ ([APS] II). Hence the assertion of Theorem 5.2.1 follows from (37), (43), (46).

We prove Lemma 5.3.1. The Galois group $\boldsymbol{G}=\operatorname{Gal}(\tilde{\mathcal{C}} / \mathcal{C})$ of the base extension $\tilde{e} \rightarrow \mathcal{C}$ naturally acts on $\tilde{S}$ so that the restriction of this action to a neighborhood of a singular fiber of $\boldsymbol{f}$ is nothing but the action which we already discussed here. Since the action of $\boldsymbol{G}$ is free outside the singular fibers and the dummy fiber $\boldsymbol{f}^{-1}\left(P_{0}\right)$, it suffices to show that any data of $f^{-1}\left(P_{0}\right)$ does not contribute Sign $S-(1 / N)$ Sign $\tilde{\mathcal{S}}$.

Although $\boldsymbol{f}^{-1}\left(P_{0}\right)$ is a component of fixed curves of some element of $\boldsymbol{G}$, it does not contribute the integral of the equivariant L-form because the normal bundle of $f^{-1}\left(P_{0}\right)$ in $S$ is trivial. Therefore the assertion is clear.

Acknowledgement. The author thanks Professors Shigeru Takamura, Ken-ich Yoshikawa, Mikio Furuta, Kazuhiro Konno, Mizuho Ishizaka and Tadashi Tomaru for useful discussions. He also thanks the referee for useful comments. Thanks are also due to Professor Yukio Matsumoto for advice and encouragement for a long time.

\section{References}

[AA1] T. Arakawa and T. Ashikaga, Local splitting families of hyperelliptic pencils I. Tohoku Math. J. 53 (2001), 369-394; II. Nagoya Math. J. 175 (2004), 103-124. Zbl 1081.14508 MR 1844374 Zbl 1066.14031 MR 2085313417

[AW] M. Artin and G. Winters, Degenerate fibres and stable reduction of curves. Topology 10 (1971), 373-383. Zbl 0196.24403 MR 0476756428

[AE] T. Ashikaga and H. Endo, Various aspects of degenerate families of Riemann surfaces. Sugaku Exposition 19 (2006), 171-196. Zbl 05509067 MR 2041054418

[AI1] T. Ashikaga and M. Ishizaka, Classification of degenerations of curves of genus three via Matsumoto-Montesinos' theorem. Tohoku Math. J. 54 (2002), 195-226. Zbl 1094.14006 MR 1904949 420, 427, 432

[AI2] T. Ashikaga and M. Ishizaka, A geometric proof of the reciprocity law of Dedekind sum. Preprint. 444

[AK] T. Ashikaga and K. Konno, Global and local properties of pencils of algebraic curves. In Algebraic Geometry 2000 Azumino, ed. by S. Usui et al., Adv. Stud. Pure Math. 36, Math. Soc. Japan, Tokyo 2002, 1-49. Zbl 1088.14010 MR 1971511418 
[AY] T. Ashikaga and K. Yoshikawa, A divisor on the moduli space of curves associated to the signature of fibered surfaces. In Singularities - Niigata-Toyama 2007, Adv. Stud. Pure Math. 56, Math. Soc. Japan, Tokyo 2009, 1-34. 418, 449

[At] M. Atiyah, The logarithm of the Dedekind $\eta$-function. Math. Ann. 278 (1987), 335-380. Zbl 0648.58035 MR 0909232417

[AS] M. Atiyah and I. M. Singer, The index of elliptic operators III. Ann. of Math. 87 (1968), 546-604. Zbl 0164.24301 MR 0236952 452, 456

[APS] M. Atiyah, V. K. Patodi and I. M. Singer, Spectral asymmetry and Riemannian geometry, I. Math. Proc. Cambridge Philos. Soc. 77 (1975), 43-69; II. ibid. 78 (1975), 405-432. Zbl 0297.58008 MR 0397797 Zbl 0314.58016 MR 0397798 418, 447, 458

[BPV] W. Barth, C. Peters and A. Van de Ven, Compact complex surfaces. Ergeb. Math. Grenzgeb. (3) 4, Springer-Verlag, Berlin 1984. Zbl 0718.14023 MR 749574 428, 430

[Br] E. Brieskorn, Rationale Singularitäten komplexer Flächen. Invent. Math. 4 (1968), 336-358. Zbl 0219.14003 MR 0222084 430, 432

[C] C.H. Clemens, Picard-Lefschetz theorem for families of nonsingular algebraic varieties acquiring ordinary singularities. Trans. Amer. Math. Soc. 136 (1969), 93-108. Zbl 0185.51302 MR 0233814438

[DM] P. Deligne and D. Mumford, The irreducibility of the space of curves of given genus. Inst. Hautes Études Sci. Publ. Math. 36 (1969), 75-110. Zbl 0181.48803 MR 0262240 419, 428

[E] H. Endo, Meyer's signature cocycle and hyperelliptic fibrations (with an appendix by T. Terasoma). Math. Ann. 316 (2000), 237-257. Zbl 0948.57013 MR 1741270417

[Fu] M. Furuta, Surface bundle and local signature. In The report of the symposium of Topology related to Riemann surfaces, ed. by N. Kawazumi, 1999, 47-53 (in Japanese). 447

[GH] P. Griffiths and J. Harris, Principles of algebraic geometry. John Wiley and Sons Inc., New York 1978. Zbl 0408.14001 MR 0507725456

[Gr] H. Grauert, Über Modifikationen und exzeptionelle analytische Mengen. Math. Ann. 146 (1962), 331-368. Zbl 0173.33004 MR 0137127429

[HM] J. Harris and D. Mumford, On the Kodaira dimension of the moduli space of curves. Invent. Math. 67 (1982), 23-88. Zbl 0506.14016 MR 0664324

[Ha] J. Harvey, Cyclic groups of automorphisms of a compact Riemann surface. Quart. J. Math. Oxford Ser. (2) 17 (1966), 86-97. Zbl 0156.08901 MR 0201629433

[Hi1] F. Hirzebruch, Neue Topologische Methoden in der Algebraischen Geometrie. Ergeb. Math. Grenzgeb. 9, Springer-Verlag, Heidelberg 1956. Zbl 0070.16302 MR 0082174 418

[Hi2] F. Hirzebruch, The signature theorem: reminiscences and recreation. In Prospects in Mathematics, Ann. Math. Stud. 70, Princeton University Press, Princeton, N.J., 1971, 3-31. Zbl 0252.58009 MR 0368023418

[HZ] F. Hirzebruch and D. B. Zagier, The Atiyah-Singer theorem and elementary number theory. Math. Lecture Ser. 3, Publish or Perish, Boston, Mass., 1974. Zbl 0288.10001 MR 0650832 444, 456 
[Hol] R. -P. Holzapfel, Chern number relations for locally abelian Galois coverings of algebraic surfaces. Math. Nachr. 138 (1988), 263-292. Zbl 0668.14009 MR 0975215 419, 445

[Ii] S. Iida, Adiabatic limit of $\eta$-invariants and the Meyer function of genus two. Preprint, http://kyokan.ms.u-tokyo.ac.jp/users/preprint/pdf/2007-11.pdf 417

[Im] Y. Imayoshi, Holomorphic families of Riemann surfaces and Teichmüller spaces. In Riemann surfaces and related topics, Ann. of Math. Stud. 97, Princeton University Press, Princeton, N.J.; University of Tokyo Press, Tokyo 1981, 277-300. Zbl 0476.32025 MR 0624799429

[Ka] T. Kawasaki, The signature theorem for V-manifolds. Topology 17 (1978), 75-83. Zbl 0392.58009 MR 0474432 419, 453

[Ku] Y. Kuno, The mapping class group and the Meyer function for plane curves. Math. Ann. 342 (2008), 923-949. Zbl 1162.57018 MR 2443768418

[Ma1] Y. Matsumoto, On 4-manifolds fibered by tori. Proc. Japan Acad. 58 (1982), 298-301; II, ibid. 59 (1983), 100-103. Zbl 0515.55012 MR 0682687 Zbl 0532.55020 MR 0711307 417

[Ma2] Y. Matsumoto, Lefschetz fibrations of genus two - a topological approach. In Topology and Teichmüller spaces, World Scientific Publishing, River Edge, NJ, 1996, 123-148 1996. Zbl 0921.57006 MR 1659687417

[MM1] Y. Matsumoto and J. M. Montesinos-Amilibia, Pseudo-periodic maps and degeneration of Riemann surfaces I, II. Preprints, University of Tokyo and Universidad Complutense de Madrid, 1991/1992. 419, 420, 421, 422, 423, 429, 433, 438

[MM2] Y. Matsumoto and J. M. Montesinos-Amilibia, Pseudo-periodic homeomorphisms and degeneration of Riemann surfaces. Bull. Amer. Math. Soc. 30 (1994), 70-75. Zbl 0797.30036 MR 1217354 423, 425, 427

[Mo] T. Morifuji, On Meyer's function of hyperelliptic mapping class groups. J. Math. Soc. Japan 55 (2003), 117-129. Zbl 1031.57017 MR 1939188417

$[\mathrm{Mu}]$ D. Mumford, The topology of normal singularities of an algebraic surface and a criterion for simplicity. Inst. Hautes Études Sci. Publ. Math. 9 (1961), 5-22. Zbl 0108.16801 MR 0153682432

[My] G. Myerson, On semi-regular finite continued fractions. Arch. Math. 48 (1987), 420-425. Zbl 0596.10009 MR 0888871 419, 445

[NU] Y. Namikawa and K. Ueno, The complete classification of fibres in pencils of genus two. Manuscripta Math. 9 (1973), 143-186. Zbl 0263.14007 MR 0369362 434, 435

[N1] J. Nielsen, Die Struktur periodischer Transformation von Flächen. Mat.-Fys. Medd. Danske Vid. Selsk. 15 (1937); Jakob Nielsen: collected mathematical papers, Vol. 2, Contemp. Mathematicians, Birkhäuser, Boston, Mass., 1986. 418, 421

[N2] J. Nielsen, Surface transformation classes of algebraically finite type. Mat.-Fys. Medd. Danske Vid. Selsk. 21 (1944), 3-89; Jakob Nielsen: collected mathematical papers, Vol. 2, Contemp. Mathematicians, Birkhäuser, Boston, Mass., 1986. Zbl 0063.05952 MR 0015791 418, 421

[OS] S. Ogata and M. Saito, Signature defects and eta functions of degenerations of abelian varieties. Japan. J. Math. 23, 319-364. Zbl 0895.58051 MR 1486517 
[R] O. Riemenschneider, Dihedral singularities: Invariants, equations and infinitesimal deformations. Bull. Amer. Math. Soc. 82 (1978), 745-747. Zbl 0332.32006 MR 0407316 430

[Sa] I. Satake, The Gauss-Bonnet theorem for V-manifolds. J. Math. Soc. Japan 9 (1957), 464-492. Zbl 0080.37403 MR 0095520 452

[Sm] I. Smith, Lefschetz fibrations and the Hodge bundle. Geom. Topol. 3 (1999), 211-233. Zbl 0929.53047 MR 1701812418

[Tak] S. Takamura, Towards the classification of atoms of degenerations II. Cyclic quotient construction of degenerations of complex curves. Res. Inst. Math. Sci. Kyoto Univ. Preprint Ser. No. 1344, 2001. 419, 420, 423, 425, 436, 438, 441

[Tan] S. L. Tan, On the invariants of base changes of pencils of curves I. Manuscripta Math. 84 (1994), 225-244; II. Math. Z. 222 (1996), 655-676. Zbl 0835.14012 MR 1291119 Zbl 0864.14016 MR 1406272 418

[Te1] T. Terasoma, Degeneration of curves and analytic deformations. Preprint, 1998. 419

[Te2] T. Terasoma, An appendix to Endo's paper. Math. Ann. 316 (2000), 355-357. 417

[To] T. Tomaru, Pencil genus for normal surface singularities. J. Math. Soc. Japan 59 (2007), 35-80. Zbl 1140.32016 MR 2302662 425

[U] K. Ueno, Discriminants of curves of genus 2 and arithmetic surfaces. In Algebraic geometry and commutative algebra, Volume II, Kinokuniya, Tokyo 1988, 749-770. Zbl 0707.14025 MR 0977781417

[V] E. Viehweg, Canonical divisors and the additivity of the Kodaira dimension for morphisms of relative dimension one. Compositio Math. 35 (1977), 197-233; Corrigendum. ibid. 35 (1977), 197-223. Zbl 0357.14014 MR 0569690 Zbl 0371.14021 MR 0569696 418

[X] G. Xiao, The fibrations of algebraic surfaces. Shanghai Scientific and Technical Publishers, 1992 (in Chinese). 417

Received April 25, 2008

Tadashi Ashikaga, Faculty of Engineering, Tohoku Gakuin University, Tagajo, Miyagi, 985-8537, Japan

E-mail: tashikaga@tjcc.tohoku-gakuin.ac.jp 